\title{
Andreas Burth
}

\section{Der Effekt der Größe kommunaler Volksvertretungen auf die Kommunalfinanzsituation}

\author{
Haushaltskonsolidierung; Kommunalfinanzen; Kreistag; Rat
}

Bedingt durch die kritische Lage der kommunalen Finanzen sind Haushaltskonsolidierungsmaßnahmen in vielen Kommunen dringend geboten. Der Bund der Steuerzahler Hessen schlägt in diesem Kontext vor, kommunale Volksvertretungen zu verkleinern. Der vorliegende Beitrag nimmt eine Bestandsaufnahme hinsichtlich der Kommunalfinanzsituation sowie der Größe kommunaler Volksvertretungen vor und analysiert die Einsparpotentiale, die durch Verkleinerungen realisiert werden können. Im Ergebnis wird festgestellt, dass sich im Fall der Räte (Städte/Gemeinden) durch eine Verkleinerung sowie im Fall der Kreistage (Landkreise) durch eine Vergrößerung der Sitzzahlen Konsolidierungspotentiale erschließen lassen.

\section{Einleitung und Zielsetzung}

Die kommunalen Finanzen befinden sich in einer kritischen Lage (Anton/Diemert 2009); (Anton/ Diemert 2010); (Deutscher Landkreistag 2011); (Deutscher Städte- und Gemeindebund 2011). Durch die Finanzkrise hat sich die Situation weiter verschlechtert. Viele Kommunen haben auch in konjunkturell guten Zeiten ihre Verschuldung nicht wesentlich zurückgeführt und sehen sich in der Krise nun einem hohen Schuldendienst einerseits sowie sinkenden Einnahmen und steigenden Ausgaben andererseits konfrontiert. In Anbetracht dieser Konstellation sind entsprechende Konsolidierungsmaßnahmen dringend geboten.

Für eine Konsolidierung der kommunalen Haushalte sind in erster Linie die jeweiligen Kommunen selbst verantwortlich, indem sie ihre Spielräume im Hinblick auf große wie auch kleine Einnahmeerhöhungs- und Ausgabensenkungspotentiale ausloten. Werden diese Maßnahmen nicht unternommen, droht die Zahlungsunfähigkeit. Zwar gilt in diesem Fall die Einstandspflicht der Länder (Nierhaus/Gebhardt 1999); (Rehm/Tholen 2008, S. 80); (Zimmermann 2009, S. 201), jedoch sollte dies nicht als Ansporn zur unverantwortlichen Haushalts- und Finanzpolitik interpretiert werden.

Die Einstandspflicht der Länder verdeutlicht gleichwohl deren Interesse an stabilen Kommunalfinanzen. Von der Landesebene ausgehende Konsolidierungsanreize können u. a. über die Ausgestaltung des Kommunalrechts gesetzt werden. Einen solchen Anreiz hat das Land Hessen etabliert, indem es in die Kommunalverfassung ein Optionsrecht zur freiwilligen Verkleinerung der 
kommunalen Volksvertretungen ${ }^{1}$ integriert hat. Der hessische Bund der Steuerzahler hat die Kommunen in Hessen aufgefordert, dieses Optionsrecht wahrzunehmen, da dadurch ein Beitrag zur Haushaltskonsolidierung geleistet werden könne (Bund der Steuerzahler Hessen e. V. 2010 a; Bund der Steuerzahler Hessen e. V. 2010 b). Auch die bisherige Forschung zu staatlichen Volksvertretungen suggeriert, dass große Parlamente mehr ausgeben als kleinere Parlamente (Gilligan/Matsusaka 2001); (Gilligan/Matsusaka 1995). Für die kommunale Ebene liegen demgegenüber keine empirischen Forschungsergebnisse zu den Auswirkungen auf die Haushalts- und Finanzlage vor. ${ }^{2}$

Vor diesem Hintergrund stellt sich die Frage, inwieweit bezüglich der kommunalen Volksvertretungen, d. h. den kommunalen Hauptorganen, tatsächlich Einsparpotentiale realisiert werden können. Ziel des vorliegenden Beitrags ist es daher, zunächst eine vergleichende Analyse im Hinblick auf die Kommunalfinanzsituation und die Größe der Kommunalparlamente in den einzelnen Ländern vorzunehmen. Aufbauend hierauf wird untersucht, inwiefern ein Kausalzusammenhang zwischen der kommunalen Volksvertretungsgröße und der aktuellen Kommunalfinanzsituation besteht.

\section{Stand der Forschung}

Zur Bestimmung des aktuellen Forschungsstandes ist vom Autor im Juli 2011 eine Literaturrecherche durchgeführt worden. Ausgewählte, relevante Forschungsarbeiten werden in Tabelle 1 kurz vorgestellt, um darauf aufbauend bestehende Forschungslücken aufzuzeigen.

\begin{tabular}{|l|l|l|l|}
\hline Jahr & $\begin{array}{l}\text { Au- } \\
\text { tor(en) }\end{array}$ & Titel & Inhalt/Ergebnisse \\
\hline 1989 & $\begin{array}{l}\text { Muzzio/ } \\
\text { Tomp- } \\
\text { kins }\end{array}$ & $\begin{array}{l}\text { On the Size of the City } \\
\text { Council: Finding the } \\
\text { Mean }\end{array}$ & $\begin{array}{l}\text { Muzzio/Tompkins untersuchen die durchschnittliche Größe } \\
\text { städtischer Volksvertretungen in den USA. Im Hinblick auf } \\
\text { den Zusammenhang von Größe und Finanzlage stellen die } \\
\text { Autoren die These auf, dass die Größe von Räten im Span- } \\
\text { nungsfeld von Effizienz und der Verfügbarkeit von Alter- } \\
\text { nativen steht. }\end{array}$ \\
\hline 1991 & Yates & $\begin{array}{l}\text { A House of Our Own or } \\
\text { A House We've Out- } \\
\text { grown? An Argument } \\
\text { for Increasing the Size } \\
\text { of the House of Repre- } \\
\text { sentatives }\end{array}$ & $\begin{array}{l}\text { Der Beitrag von Yates zielt darauf ab zu untersuchen, ob } \\
\text { das House of Representatives der USA vor dem Hintergrund } \\
\text { demokratischer Grundsätze zu klein oder zu groß ist. Yates } \\
\text { schlägt als Ergebnis seiner Analyse eine Vergrößerung vor. }\end{array}$ \\
\hline
\end{tabular}

1 Die Volksvertretungen der Städte und Gemeinden werden i. d. R. als „Stadtrat“ bzw. „Gemeinderat“ (oder kurz „Rat") bezeichnet. Je nach Bundesland sind jedoch z. T. auch andere Bezeichnungen üblich (z. B. „Gemeindevertretung“ bzw. „Stadtverordnetenversammlung“ in Hessen). Im weiteren Verlauf des Beitrags wird die Kurzbezeichnung „Rat“ verwendet. Auf Ebene der Landkreise wird in allen Flächenländern vom „Kreistag“ gesprochen. Die Volksvertretung der bayerischen Bezirke heißt „Bezirkstag“. Da es sich hierbei um eine bayerische Besonderheit handelt, sind Bezirke nicht Gegenstand der vorliegenden Untersuchung.

2 Siehe hierzu Abschnitt II. 


\begin{tabular}{|c|c|c|c|}
\hline 1995 & $\begin{array}{l}\text { Crain/ } \\
\text { Muris }\end{array}$ & $\begin{array}{l}\text { Legislative Organiza- } \\
\text { tion of Fiscal Policy }\end{array}$ & $\begin{array}{l}\text { Crain/Muris untersuchen den Einfluss der Organisation der } \\
\text { Legislative (insb. bzgl. Ausschüssen) auf fiskalische Ent- } \\
\text { scheidungen. Im Ergebnis wird festgestellt, dass Ausgaben } \\
\text { höher sind, wenn mehr als ein Ausschuss die Ausgabenho- } \\
\text { heit hat. Ferner wird die Hypothese unterstützt, dass, wenn } \\
\text { ein Ausschuss neben der Ausgabenhoheit auch die Hoheit } \\
\text { über Steuerentscheidungen hat, ein größerer Anreiz zur Be- } \\
\text { steuerung besteht, als wenn mehrere Ausschüsse zuständig } \\
\text { sind. }\end{array}$ \\
\hline 1997 & Cusack & $\begin{array}{l}\text { Partisan politics and } \\
\text { public finance: } \\
\text { Changes in public } \\
\text { spending in the indus- } \\
\text { trialized democracies, } \\
\text { 1955-1989 }\end{array}$ & $\begin{array}{l}\text { Cusack evaluiert den Effekt von Parteipolitik auf Verände- } \\
\text { rungen im Ausgabenniveau der öffentlichen Einheiten. Ab- } \\
\text { gedeckt werden } 16 \text { OECD-Länder. Im Ergebnis stellt der } \\
\text { Autor fest, dass die Ideologie der regierenden Partei(en) und } \\
\text { die Distanz zwischen der Ideologie der regierenden Par- } \\
\text { tei(en) und der Wählerschaft Einfluss auf die Ausgabenpo- } \\
\text { litik hat. }\end{array}$ \\
\hline 2001 & $\begin{array}{l}\text { Gilligan/ } \\
\text { Matsusa- } \\
\text { ka }\end{array}$ & $\begin{array}{l}\text { Fiscal Policy, Legisla- } \\
\text { ture Size, and Political } \\
\text { Parties: Evidence from } \\
\text { State and Local Gov- } \\
\text { ernments in the First } \\
\text { Half of the 20th Century }\end{array}$ & $\begin{array}{l}\text { Der Beitrag analysiert, ob die Fiskalpolitik von Staat und } \\
\text { Kommunen in der ersten Hälfte des 20. Jahrhunderts von } \\
\text { der Anzahl der Sitze der jeweiligen staatlichen Gebietskör- } \\
\text { perschaft abhängen. Es wird festgestellt, dass große staatli- } \\
\text { che Volksvertretungen höhere Ausgaben haben als kleine. } \\
\text { Ferner wird festgestellt, dass lediglich die Größe des Ober- } \\
\text { hauses, nicht aber die des Unterhauses, Einfluss auf die Fis- } \\
\text { kalpolitik hat. }\end{array}$ \\
\hline 2004 & $\begin{array}{l}\text { Petters- } \\
\text { son- } \\
\text { Lidbom }\end{array}$ & $\begin{array}{l}\text { Does the size of the leg- } \\
\text { islature affect the size of } \\
\text { government? Evidence } \\
\text { from two natural exper- } \\
\text { iments }\end{array}$ & $\begin{array}{l}\text { Der Autor untersucht den kausalen Effekt der Größe einer } \\
\text { Volksvertretung auf die Größe der Regierung/Verwaltung. } \\
\text { Es wird ein negativer Kausalzusammenhang identifiziert. }\end{array}$ \\
\hline 2008 & $\begin{array}{l}\text { Auriol/ } \\
\text { Gary- } \\
\text { Bobo }\end{array}$ & $\begin{array}{l}\text { On the Optimal Number } \\
\text { of Representatives }\end{array}$ & $\begin{array}{l}\text { Auriol/Gary-Bobo entwickeln eine Theorie der optimalen } \\
\text { Anzahl von Volksvertretern einer Gebietskörperschaft. Auf } \\
\text { Basis ökonometrischer Tests kommen die Autoren zu dem } \\
\text { Ergebnis, dass große Volksvertretungen in einer positiven } \\
\text { Korrelationsbeziehung zu den Indikatoren Bürokratie, Bar- } \\
\text { rieren für Unternehmertum und wahrgenommene Korrup- } \\
\text { tion stehen. }\end{array}$ \\
\hline 2009 & $\begin{array}{l}\text { Heine- } \\
\text { mann } \\
\text { u. a. }\end{array}$ & $\begin{array}{l}\text { Der kommunale Kas- } \\
\text { senkredit zwischen Li- } \\
\text { quiditätssicherung und } \\
\text { Missbrauchsgefahr }\end{array}$ & $\begin{array}{l}\text { Heinemann u. a. untersuchen die Determinanten der Ver- } \\
\text { schuldung in Deutschland (insb. auch der Kassenkreditver- } \\
\text { schuldung). Im Ergebnis wird festgestellt, dass u. a. die Be- } \\
\text { völkerungsdichte, die Gesamteinnahmenquote, die Schlüs- } \\
\text { selzuweisungsquote und die Gewerbesteuerquote (Quoten } \\
\text { jeweils in \% des BIP) einen signifikanten Einfluss auf die } \\
\text { Kassenkreditverschuldung haben. }\end{array}$ \\
\hline 2011 & $\begin{array}{l}\text { Le Maux/ } \\
\text { Rocaboy/ } \\
\text { Good- } \\
\text { speed }\end{array}$ & $\begin{array}{l}\text { Political fragmentation, } \\
\text { party ideologiy and } \\
\text { public expenditures }\end{array}$ & $\begin{array}{l}\text { In diesem Beitrag wird analysiert, ob die politische Macht } \\
\text { (v. a. determiniert durch die Anzahl der Sitze im Parlament) } \\
\text { der Koalition Einfluss auf die Ausgabenhöhe hat. Ergebnis } \\
\text { ist, dass der Stimmenvorsprung und die Fragmentierung } \\
\text { von Regierungs- und Oppositionsparteien die Ausgabenpo- } \\
\text { litik beeinflussen. }\end{array}$ \\
\hline
\end{tabular}

Tabelle 1: Ausgewählte Forschungsarbeiten zur Volksvertretungsgröße und Kommunalfinanzsituation

Quelle: Eigene Darstellung 
Wie aus Tabelle 1 ersichtlich wird, deckt die bisherige, wissenschaftliche Forschung v. a. die Ebene der staatlichen Volksvertretungen ab (Crain/Muris 1995); (Le Maux/Rocaboy/Goodspeed 2011); (Gilligan/Matsusaka 2001). Die vorliegenden Untersuchungen beschränken sich darüber hinaus auf die Auswirkungen auf die Ausgabenseite, ohne jedoch explizit andere Indikatoren für die Haushalts- und Finanzlage (z. B. Verschuldung, Haushaltsausgleich, Einnahmepositionen) zu betrachten (Cusack 1997); (Gilligan/Matsusaka 2011); (Le Maux/Rocaboy/Goodspeed 2011). Ferner berücksichtigt die empirische Forschung zur optimalen Größe von Volksvertretungen nur begrenzt die Auswirkungen auf die Haushalts- und Finanzlage (Auriol/Gary-Bobo 2008). Die Untersuchung von Muzzio/Tompkins hat zwar kommunale Vertretungen zum Gegenstand, gleichwohl hat der Beitrag primär deskriptiven Charakter. Im Hinblick auf den Zusammenhang zwischen der Größe von Volksvertretungen und der Kommunalfinanzsituation werden lediglich Thesen aufgestellt, aber nicht getestet (Muzzio/Tompkins 1989). Heinemann u. a. berücksichtigen ihrerseits die kommunale Volksvertretungsgröße nicht als möglichen Einflussfaktor (unabhängige Variable) auf die Kassenkreditbestände. Ferner wird die Verschuldung als abhängige Variable betrachtet, nicht jedoch eine Saldogröße, wie z. B. der Haushaltssaldo (Heinemann u. a. 2009). Der vorliegende Beitrag soll diese Forschungslücke schließen und untersuchen, inwiefern Verkleinerungen einer kommunalen Volksvertretung zu einer Verbesserung der Kommunalfinanzsituation beitragen können.

\section{Analyse der Kommunalfinanzsituation}

Das Statistische Bundesamt veröffentlicht regelmäßig finanzstatistische Daten zu den Kommunalfinanzen. Den publizierten Finanzstatistiken zufolge, hat sich die Situation der Kommunalfinanzen im Zuge der Finanz- und Wirtschaftskrise stark verschlechtert. Dies wird v. a. am kommunalen Finanzierungssaldo ${ }^{3}$ deutlich.

Während der kommunale Finanzierungssaldo der Flächenländer ${ }^{4}$ in den Jahren unmittelbar vor der Krise (2007 und 2008) mit 8.175 Mio. Euro bzw. 8.352 Mio. Euro deutlich positiv gewesen ist (Statistisches Bundesamt 2009); (Statistisches Bundesamt 2010 a), hat sich das Vorzeichen 2009 umgedreht: Im ersten von der Finanzkrise beeinträchtigten Haushaltsjahr beläuft sich der kommunale Finanzierungssaldo auf -7.176 Mio. Euro (Statistisches Bundesamt 2010 b). Im Jahr 2010 hat sich die Situation um zusätzliche 541 Mio. Euro auf -7.717 Mio. Euro verschlechtert (Statistisches Bundesamt 2011). Zwischen den Ländern bestehen indes große Unterschiede (siehe Tab. 2). Den schlechtesten Wert je Einwohner weist das Land Hessen aus. Der dortige (negative) Saldo je Einwohner (-437,16 Euro je Einwohner) ist betragsmäßig mehr als doppelt so hoch wie der Finanzierungssaldo des Bundeslandes mit dem zweitschlechtesten kommunalen Finanzierungssaldo (Nordrhein-Westfalen: -218,42 Euro je Einwohner). Ein Grund für das außerordentlich schlechte Abschneiden Hessens ist in der starken Abhängigkeit von der Gewerbesteuer zu finden,

3 Der kommunale Finanzierungssaldo ist eine stark aggregierte Kennzahl zur Beurteilung der kommunalen Finanzsituation. Er berechnet sich über die Summe aus dem Saldo der bereinigten Ausgaben und Einnahmen und dem Saldo der haushaltstechnischen Verrechnungen (Statistisches Bundesamt 2011); (Burth u. a. 2012, S. 142).

$4 \mathrm{Im}$ vorliegenden Beitrag werden aufgrund nicht vergleichbarer Einnahme-, Ausgabe- und Aufgabenstrukturen nur die Kommunen der Flächenländer, nicht jedoch die Stadtstaaten, untersucht. 
welche besonders stark von der Finanz- und Wirtschaftskrise betroffen ist (Burth/Hilgers 2011). Eine weitere Ursache liegt im hessischen Konjunkturpaket begründet. Bei den investierten Mittel handelt es sich zwar in letzter Instanz um Landesmittel, jedoch belasten sie den kommunalen Finanzierungssaldo, da die von den Kommunen getätigten Investitionen erst über einen Zeitraum von 30 Jahren vom Land zurückerstattet werden (Hessisches Ministerium der Finanzen 2008, S. 3).

\begin{tabular}{|c|c|c|c|c|}
\hline \multirow[t]{2}{*}{ Flächenland } & \multicolumn{2}{|c|}{ Kommunaler Finanzierungssaldo } & \multirow{2}{*}{$\begin{array}{l}\text { Kommunale } \\
\text { Verschuldung } \\
\text { je Einwohner } \\
\text { (in Euro) }\end{array}$} & \multirow{2}{*}{$\begin{array}{c}\text { Einwohner } \\
\text { (zum } \\
\text { 30.6.2009) }\end{array}$} \\
\hline & $\begin{array}{c}\text { gesamt } \\
\text { (in Mio. Euro) }\end{array}$ & $\begin{array}{l}\text { je Einwohner } \\
\text { (in Euro) }\end{array}$ & & \\
\hline Baden-Württemberg & -688 & $-64,01$ & 967,63 & 10.747 .905 \\
\hline Bayern & -340 & $-27,21$ & $1.359,20$ & 12.497 .082 \\
\hline Brandenburg & -109 & $-43,33$ & $1.100,31$ & 2.515 .679 \\
\hline Hessen & -2.649 & $-437,16$ & $2.775,43$ & 6.059 .581 \\
\hline Mecklenburg-Vorpommern & 39 & 23,54 & $1.830,05$ & 1.656 .761 \\
\hline Niedersachsen & -596 & $-75,01$ & $1.785,52$ & 7.945 .244 \\
\hline Nordrhein-Westfalen & -2.382 & $-133,12$ & $2.697,10$ & 17.893 .212 \\
\hline Rheinland-Pfalz & -692 & $-172,19$ & $2.795,60$ & 4.018 .855 \\
\hline Saarland & -224 & $-218,42$ & $2.614,18$ & 1.025 .531 \\
\hline Sachsen & 211 & 50,51 & $1.089,65$ & 4.177 .393 \\
\hline Sachsen-Anhalt & 56 & 23,65 & $1.724,33$ & 2.367 .554 \\
\hline Schleswig-Holstein & -323 & $-114,13$ & $1.315,33$ & 2.830 .118 \\
\hline Thüringen & -20 & $-8,86$ & $1.201,22$ & 2.257 .063 \\
\hline Flächenländer & -7.717 & $-101,55$ & $1.861,17$ & 75.991 .978 \\
\hline
\end{tabular}

Tabelle 2: Kommunaler Finanzierungssaldo für das Jahr 2010 sowie kommunale Verschuldung zum 31.12.2009 nach Flächenländern

Quelle: Eigene Darstellung; Daten entnommen aus Statistisches Bundesamt 2011; Statistisches Bundesamt 2010 c; berücksichtigt werden folgende Schuldenarten: Kreditmarktschulden, Schulden bei öffentlichen Haushalten, kreditähnliche Rechtsgeschäfte, innere Schulden, Kassenverstärkungskredite, Bürgschaften, Garantien und sonstige Gewährleistungen.

Die höchsten Finanzierungssalden sind in den ostdeutschen Flächenländern zu beobachten. Die Länder Mecklenburg-Vorpommern, Sachsen und Sachsen-Anhalt können als einzige Bundesländer 2010 einen positiven kommunalen Finanzierungssaldo ausweisen. Ein Hauptgrund für die vergleichsweise gute Situation der neuen Länder liegt darin, dass die laufenden Zuweisungen im Osten eine deutlich wichtigere Einnahmequelle darstellen als dies im Westen der Fall ist. Die westdeutschen Kommunen finanzieren sich demgegenüber erheblich stärker aus Steuerquellen (insb. Gewerbesteuer, gemeindlicher Einkommensteueranteil) (Anton/Diemert 2010, S. 6). Die Steuereinnahmen sind im Zuge der Finanz- und Wirtschaftskrise wesentlich stärker zurückgegangen als die laufenden Zuweisungen von Land/Bund (Anton/Diemert 2009, S. 5 ff.); (Anton/ Diemert 2010, S. 5 ff.). 
Ein temporär negativer kommunaler Finanzierungssaldo stellt jedoch nicht notwendigerweise ein Problem dar. Sofern Kommunen in guten Zeiten sparen (d. h. Rücklagen aufbauen), können sie in Krisenzeiten diese Rücklagen aufbrauchen bzw. notfalls Kredite aufnehmen. Dieses Verhalten ist in der Praxis jedoch nur in wenigen Kommunen beobachtbar. Dies hat zur Folge, dass viele Kommunen auch in Krisenzeiten erhebliche Finanzmittel für den Schuldendienst (Zins und Tilgung) aufwenden müssen, was den Haushaltsausgleich erschwert. 2008 waren nur 2.382 der 13.436 deutschen Kommunen (17,73\%) unter Berücksichtigung der Kassenkredite, Kreditmarktschulden, Schulden bei öffentlichen Haushalten und kreditähnlichen Rechtsgeschäfte der Kernverwaltung und der Schulden der rechtlich unselbstständigen Eigenbetriebe faktisch schuldenfrei (Gnädinger 2010, S. 113 ff.).

Zwischen den Ländern bestehen bezüglich der kommunalen Verschuldungssituation erhebliche Unterschiede (siehe Tab. 2). So weisen die Kommunen der Länder Baden-Württemberg, Sachsen und Brandenburg die niedrigsten Schulden pro Kopf aus. „Spitzenreiter“ in der pro-Kopf-Verschuldung sind die Kommunen der Länder Rheinland-Pfalz, Hessen, Nordrhein-Westfalen und Saarland. Es fällt ferner auf, dass mit Saarland, Rheinland-Pfalz, Nordrhein-Westfalen und Hessen gleichzeitig diejenigen Länder die höchste Kommunalverschuldung je Einwohner ausweisen, die auch betragsmäßig hohe negative kommunale Finanzierungssalden je Einwohner verzeichnen. Umgekehrt stehen Länder mit niedriger Kommunalverschuldung je Einwohner (z. B. Sachsen, Bayern) beim kommunalen Finanzierungssaldo vergleichsweise gut dar. ${ }^{5}$ Dies verdeutlicht den Einfluss der infolge der Verschuldung zu leistenden Zinsausgaben auf den kommunalen Finanzierungssaldo. $^{6}$

Die beiden oben erläuterten Kennzahlen (Finanzierungssaldo und Schuldenstand) geben einen grundsätzlichen Einblick in die Finanzlage der Kommunen. Die finanzielle Situation der Kommunen können die beiden Kennzahlen aufgrund des Fokus auf den Kernhaushalt sowie der fehlenden Ressourcenverbrauchsorientierung jedoch nicht vollständig abbilden. Hintergrund hierfür ist, dass die Finanzstatistik noch auf kameralen Daten basiert und damit aktuell weder doppische Salden (z. B. ordentliches Jahresergebnis) noch doppische Bilanzkennzahlen (z. B. Fremd-/Eigenkapital) ${ }^{7}$ für die Kernverwaltung beinhaltet (Statistisches Bundesamt 2010 c); (Statistisches Bundesamt 2011). Auch konsolidierte Finanzdaten unter Berücksichtigung der Auslagerungen (im Sinne des Gesamtabschlusses) ${ }^{8}$ werden derzeit noch nicht finanzstatistisch erfasst (Gnädinger 2011, S. 63 ff.).

5 Unterschiede in der Verschuldungshöhe bestehen sowohl für die Summe aller Schuldenarten als auch für einzelne Schuldenarten. So existieren beispielsweise in Nordrhein-Westfalen, Rheinland-Pfalz und dem Saarland vergleichsweise hohe Kassenkreditbestände, während diese Schuldenart in Baden-Württemberg, Bayern und Sachsen im Landesdurchschnitt nur geringe Volumina erreicht (Statistisches Bundesamt $2010 \mathrm{c}$ ). Gnädinger sieht unterschiedlich restriktive Kassenkreditschuldenbremsen im Haushaltsrecht als einen Grund für Unterschiede im Kassenkreditniveau (Gnädinger 2011). Heinemann u. a. identifizieren demgegenüber z. B. die Bevölkerungsdichte, die Gesamteinnahmenquote, die Schlüsselzuweisungsquote und die Gewerbesteuerquote (Quoten jeweils in \% des BIP) als signifikante Determinanten der Kassenkreditverschuldung (Heinemann u. a. 2009, S. 71 ff.).

6 Es sei gleichwohl angemerkt, dass die Zinsausgaben nur eine von mehreren Komponenten in der Berechnungsformel des kommunalen Finanzierungssaldos darstellen. Weitere wichtige Faktoren sind z. B. die Personalausgaben und die Steuereinnahmen.

7 Zur Diskussion um die Aussagekraft des Indikators „Eigenkapital“ siehe Budäus/Hilgers 2010 a; Mühlenkamp/ Magin 2010.

8 Zur Bedeutung des Gesamtabschlusses siehe Budäus/Hilgers 2010 b; Müller-Marqués Berger/Krebs 2010. 


\section{Vergleichende Analyse der Größe kommunaler Volksvertretungen}

\section{Zusammensetzung der Räte}

Aufbauend auf der Analyse der Kommunalfinanzsituation soll im weiteren Verlauf eine Bestandsaufnahme hinsichtlich der Zusammensetzung der Räte in den einzelnen Flächenländern durchgeführt werden. Die Datenerhebung zur landesspezifischen Zusammensetzung des Rates ist anhand der Kommunalverfassungen/-wahlgesetze der einzelnen Länder vorgenommen worden (siehe Tab. 3). ${ }^{9}$

\begin{tabular}{|c|c|c|c|c|}
\hline Bundesland & Rechtsgrundlage & $\begin{array}{c}\text { Anzahl } \\
\text { Grö- } \\
\text { Ben- } \\
\text { klassen }\end{array}$ & $\begin{array}{l}\text { Optionsrecht zur } \\
\text { freiwilligen Ver- } \\
\text { kleinerung? }\end{array}$ & $\begin{array}{l}\text { Trennung: } \\
\text { kreisfrei vs. } \\
\text { kreisangehö- } \\
\text { rig? }\end{array}$ \\
\hline $\begin{array}{l}\text { Baden- } \\
\text { Württemberg }\end{array}$ & $\begin{array}{l}\S 25 \mathrm{Abs} .2, \S 4 \text { Abs. } 2 \text { Gemeindeordnung } \\
\text { für Baden-Württemberg }\end{array}$ & 11 & $\begin{array}{l}\text { Ja, durch Mehr- } \\
\text { heitsbeschluss }\end{array}$ & Nein \\
\hline Bayern & $\begin{array}{l}\text { Art. } 31 \text { Abs. } 2 \text { Gemeindeordnung für den } \\
\text { Freistaat Bayern }\end{array}$ & 13 & Nein & Nein \\
\hline Brandenburg & $\begin{array}{l}\S 6 \text { Abs. } 2 \text { Nr. } 1 \text { und } 2 \text { Gesetz über die } \\
\text { Kommunalwahlen im Land Brandenburg }\end{array}$ & 13 & $\begin{array}{l}\text { Ja, durch Mehr- } \\
\text { heitsbeschluss }\end{array}$ & $\mathrm{Ja}$ \\
\hline Hessen & $\S 38$ Abs. 1 Hessische Gemeindeordnung & 10 & $\begin{array}{l}\text { Ja, mit } 2 / 3-\text { Mehr- } \\
\text { heit }\end{array}$ & Nein \\
\hline $\begin{array}{l}\text { Mecklenburg- } \\
\text { Vorpommern }\end{array}$ & $\begin{array}{l}\S 4 \text { Abs. } 1 \text { Kommunalwahlgesetz für das } \\
\text { Land Mecklenburg-Vorpommern }\end{array}$ & 15 & Nein & Nein \\
\hline Niedersachsen & $\begin{array}{l}\S 32 \text { Abs. } 1 \text { Niedersächsische Gemeinde- } \\
\text { ordnung }\end{array}$ & 31 & $\begin{array}{l}\text { Ja, durch Mehr- } \\
\text { heitsbeschluss }\end{array}$ & Nein \\
\hline $\begin{array}{l}\text { Nordrhein- } \\
\text { Westfalen }\end{array}$ & $\begin{array}{l}\S 3 \text { Abs. } 2 \text { a) Kommunalwahlgesetz Nord- } \\
\text { rhein-Westfalen }\end{array}$ & 11 & $\begin{array}{l}\text { Ja, durch Mehr- } \\
\text { heitsbeschluss }\end{array}$ & Nein \\
\hline $\begin{array}{l}\text { Rheinland- } \\
\text { Pfalz }\end{array}$ & $\begin{array}{l}\S 29 \text { Abs. } 2 \text { Gemeindeordnung Rheinland- } \\
\text { Pfalz }\end{array}$ & 16 & Nein & Nein \\
\hline Saarland & $\begin{array}{l}\S 32 \text { Abs. } 2 \text { Kommunalselbstverwaltungs- } \\
\text { gesetz Saarland }\end{array}$ & 7 & Nein & Nein \\
\hline Sachsen & $\begin{array}{l}\S 25 \text { Abs. } 2 \text { Gemeindeordnung für den } \\
\text { Freistaat Sachsen }\end{array}$ & 15 & $\begin{array}{l}\text { Ja, durch Mehr- } \\
\text { heitsbeschluss }\end{array}$ & Nein \\
\hline Sachsen-Anhalt & $\begin{array}{l}\text { §36 Abs. } 3 \text { Gemeindeordnung für das } \\
\text { Land Sachsen-Anhalt }\end{array}$ & 13 & Nein & Nein \\
\hline $\begin{array}{l}\text { Schleswig- } \\
\text { Holstein }\end{array}$ & $\begin{array}{l}\S 7 \mathrm{Abs} .2, \S 8 \mathrm{Nr} .1 \text { und } 2 \text { Gesetz über die } \\
\text { Wahlen in den Gemeinden } \\
\text { und Kreisen in Schleswig-Holstein }\end{array}$ & 14 & Nein & $\mathrm{Ja}$ \\
\hline Thüringen & $\S 23$ Abs. 3 Thüringer Kommunalordnung & 12 & Nein & Nein \\
\hline
\end{tabular}

Tabelle 3: Ländervergleich der Kommunalverfassungen/-wahlgesetze (Räte)

Quelle: Eigene Darstellung

9 Es sei darauf hingewiesen, dass in einigen Ländern zusätzlich Ortsteilvertretungen existieren. Im vorliegenden Beitrag werden diese jedoch aus Gründen der Vergleichbarkeit ausgeblendet. 
Tabelle 3 verdeutlicht, dass Niedersachsen mit 31 Größenklassen eine sehr feingliedrige Struktur bei der Determinierung der Zusammensetzung des Rates gewählt hat. Die grobgliedrigste Struktur ist im Saarland, dem mit Abstand kleinsten Flächenland, zu beobachten (sieben Größenklassen). Optionsrechte zur freiwilligen Verkleinerung der Zahl der Ratsmitglieder sind in den Kommunalverfassungen/-wahlgesetzen von Baden-Württemberg, Brandenburg, Hessen, Niedersachsen, Nordrhein-Westfalen und Sachsen vorgesehen. Hessen legt den Städten und Gemeinden hierbei die größten Hürden auf: Nach $\S 38$ Abs. 2 S. 5 Hessische Gemeindeordnung ist mindestens eine 2/3-Mehrheit erforderlich. In den übrigen Ländern mit Optionsrecht genügt ein Mehrheitsbeschluss. Einer Studie des Bundes der Steuerzahler Hessen zufolge haben nur 106 von 447 hessischen Städten, Gemeinden und Landkreisen (24\%) von diesem Optionsrecht Gebrauch gemacht. Die Zahl der Sitze hat sich dabei um insgesamt 630 Sitze (4,2\%) verringert (Bund der Steuerzahler Hessen e. V. 2010 a, S. 33). Zur Ausnutzung des Optionsrechts in den übrigen Ländern liegen dem Autor keine entsprechenden Studien vor.

Eine Trennung der Größenklassen in kreisfreie und kreisangehörige Städte/Gemeinden nehmen lediglich die Länder Brandenburg und Schleswig-Holstein vor.

Die heterogene Gliederungsstruktur in den Kommunalverfassungen (insb. bzgl. Anzahl der Größenklassen und Festlegung der Grenzwerte) erschwert unmittelbare Vergleiche über Landesgrenzen hinweg. Tabelle 4 veranschaulicht aus diesem Grund die Ratsmitgliederzahlen mit vereinheitlichten Größenklassen. Falls für bestimmte Größenklassen in einzelnen Ländern keine Städte/ Gemeinden existieren, sind diese nicht berücksichtigt worden, um die Durchschnittswertermittlung nicht zu verzerren. ${ }^{10}$ Zur Bestimmung des Durchschnitts ist bei Bereichsangaben aus Vereinfachungsgründen jeweils der Mittelwert verwendet worden.

\begin{tabular}{|c|c|c|c|c|c|c|c|c|c|}
\hline Flächenland & $\begin{array}{c}\text { Bis } \\
1.000 \\
\text { Einw. }\end{array}$ & $\begin{array}{c}1.001 \\
- \\
5.000 \\
\text { Einw. }\end{array}$ & $\begin{array}{c}5.001 \\
- \\
10.000 \\
\text { Einw. }\end{array}$ & $\begin{array}{c}10.001 \\
- \\
25.000 \\
\text { Einw. }\end{array}$ & $\begin{array}{c}25.001 \\
- \\
50.000 \\
\text { Einw. }\end{array}$ & $\begin{array}{c}50.001 \\
- \\
100.000 \\
\text { Einw. }\end{array}$ & $\begin{array}{c}100.001 \\
- \\
200.000 \\
\text { Einw. }\end{array}$ & $\begin{array}{c}200.001 \\
- \\
500.000 \\
\text { Einw. }\end{array}$ & $\begin{array}{c}\text { Ab } \\
\text { 500.001 } \\
\text { Einw. }\end{array}$ \\
\hline $\begin{array}{l}\text { Baden- } \\
\text { Württemberg }\end{array}$ & 8 & $10-14$ & 18 & $22-26$ & $26-32$ & 40 & $40-48$ & $48-60$ & 60 \\
\hline Bayern & 8 & $12-16$ & 20 & $24-30$ & $30-40$ & 44 & 50 & 60 & $70-80$ \\
\hline Brandenburg & $8-10$ & $10-16$ & 18 & $22-28$ & $32-40$ & $40-46$ & $46-56$ & --- & --- \\
\hline Hessen & 15 & $15-23$ & 31 & 37 & 45 & 59 & 71 & $71-81$ & 93 \\
\hline $\begin{array}{l}\text { Mecklenburg- } \\
\text { Vorpommern }\end{array}$ & $7-9$ & $11-17$ & $17-21$ & $25-29$ & $29-37$ & $43-45$ & --- & 53 & --- \\
\hline Niedersachsen & $6-8$ & $10-14$ & $15-24$ & $26-34$ & $36-40$ & $42-44$ & $46-52$ & $54-62$ & $64-66$ \\
\hline $\begin{array}{l}\text { Nordrhein- } \\
\text { Westfalen }\end{array}$ & --- & 20 & $26-32$ & $32-38$ & $38-44$ & 50 & 58 & $58-74$ & $74-90$ \\
\hline $\begin{array}{l}\text { Rheinland- } \\
\text { Pfalz }\end{array}$ & $6-12$ & $16-20$ & $22-24$ & $28-36$ & $36-44$ & $44-52$ & $56-60$ & --- & --- \\
\hline Saarland & --- & --- & 27 & $33-39$ & $39-51$ & --- & 63 & --- & --- \\
\hline Sachsen & $8-10$ & $12-16$ & 18 & $22-26$ & $26-34$ & $38-48$ & --- & $54-60$ & 60 \\
\hline
\end{tabular}

10 So sind beispielsweise die beiden obersten Größenklassen beim Land Brandenburg nicht berücksichtigt worden, da die einwohnerstärkste Stadt Brandenburgs, Potsdam, nur rund 155.000 Einwohner zählt. 
Der Effekt der Größe kommunaler Volksvertretungen auf die Kommunalfinanzsituation

\begin{tabular}{|l|c|c|c|c|c|c|c|c|c|}
\hline $\begin{array}{l}\text { Sachsen- } \\
\text { Anhalt }\end{array}$ & $4-10$ & $12-16$ & 20 & $28-36$ & $36-40$ & 50 & --- & $56-60$ & -- \\
\hline $\begin{array}{l}\text { Schleswig- } \\
\text { Holstein }\end{array}$ & $7-11$ & $11-17$ & 19 & $23-27$ & $31-39$ & $39-43$ & --- & 49 & -- \\
\hline Thüringen & $6-8$ & $12-16$ & 20 & $24-30$ & $30-36$ & 42 & 46 & 50 & --- \\
\hline Durchschnitt & 8,73 & 14,83 & 21,65 & 29,31 & 36,77 & 45,58 & 54,44 & 58,1 & 72,50 \\
\hline
\end{tabular}

Tabelle 4: Zusammensetzung des Rates in den 13 Flächenländern nach vereinheitlichten GröBenklassen

Quelle: Eigene Darstellung; Einw. = Einwohner

Als das Land mit den kleinsten Räten kann Baden-Württemberg identifiziert werden. Ebenfalls eher kleine Räte haben die Städte und Gemeinden in Mecklenburg-Vorpommern, Sachsen, Schleswig-Holstein und Thüringen. Überdurchschnittlich große Räte sind in den Ländern Hessen, Nordrhein-Westfalen, Rheinland-Pfalz und Saarland anzutreffen. Dies gilt im Besonderen für das Land Hessen, das in allen neun Einwohnergrößenklassen die mitgliederstärksten Räte aufweist. Es ist besonders bemerkenswert, dass die Kommunen in den Ländern Hessen, Nordrhein-Westfalen, Rheinland-Pfalz und Saarland die größten Räte aufweisen, da es sich hierbei um diejenigen vier Länder handelt, deren Kommunalfinanzen sich in der schlechtesten Lage aller 13 Flächenländer befinden (siehe Abschnitt III). Auf Grundlage dieser Erkenntnisse kann die These aufgestellt werden, dass mitgliederstarke Räte größere Probleme haben, den Haushalt der eigenen Kommune auszugleichen.

\section{Zusammensetzung der Kreistage}

Neben der Zusammensetzung der Räte in den Städten und Gemeinden ist auch die Zusammensetzung der Kreistage von Bedeutung für eine umfassende Bestandsaufnahme zur Größe kommunaler Volksvertretungen. Analog zu Abschnitt IV.1 ist die Datenerhebung zur Bestimmung der Zusammensetzung der Kreistage anhand der Kommunalverfassungen/-wahlgesetze der einzelnen Bundesländer vorgenommen worden. Tabelle 5 beinhaltet die wesentlichen Merkmale der jeweiligen Landesregelungen. 


\begin{tabular}{|c|c|c|c|}
\hline Bundesland & Rechtsgrundlage & $\begin{array}{l}\text { Anzahl } \\
\text { Größen- } \\
\text { klassen }\end{array}$ & $\begin{array}{c}\text { Optionsrecht } \\
\text { zur freiwilligen } \\
\text { Verkleinerung? }\end{array}$ \\
\hline $\begin{array}{l}\text { Baden- } \\
\text { Württemberg }\end{array}$ & $\begin{array}{l}\text { § } 20 \text { Abs. } 2 \text { Landkreisordnung für Baden-Würt- } \\
\text { temberg }\end{array}$ & k. A. & Nein \\
\hline Bayern & $\begin{array}{l}\text { Art. } 24 \text { Abs. } 2 \text { Landkreisordnung für den Frei- } \\
\text { staat Bayern }\end{array}$ & 3 & Nein \\
\hline Brandenburg & $\begin{array}{l}\text { § } 6 \text { Abs. } 2 \text { Nr. } 2 \text { Gesetz über die Kommunalwah- } \\
\text { len im Land Brandenburg }\end{array}$ & 3 & $\begin{array}{l}\text { Ja, durch Mehr- } \\
\text { heitsbeschluss }\end{array}$ \\
\hline Hessen & $\S 25$ Abs. 1 Hessische Landkreisordnung & 6 & $\begin{array}{l}\text { Ja, mit } 2 / 3-M e h r- \\
\text { heit }\end{array}$ \\
\hline $\begin{array}{l}\text { Mecklenburg- } \\
\text { Vorpommern }\end{array}$ & $\begin{array}{l}\text { § } 4 \text { Abs. } 1 \text { Kommunalwahlgesetz für das Land } \\
\text { Mecklenburg-Vorpommern }\end{array}$ & 2 & Nein \\
\hline Niedersachsen & § 27 Abs. 1 Niedersächsische Landkreisordnung & 10 & $\begin{array}{l}\text { Ja, durch Mehr- } \\
\text { heitsbeschluss }\end{array}$ \\
\hline $\begin{array}{l}\text { Nordrhein- } \\
\text { Westfalen }\end{array}$ & $\begin{array}{l}\S 3 \text { Abs. } 2 \text { b) Kommunalwahlgesetz Nordrhein- } \\
\text { Westfalen }\end{array}$ & 5 & $\begin{array}{l}\text { Ja, durch Mehr- } \\
\text { heitsbeschluss }\end{array}$ \\
\hline Rheinland-Pfalz & $\S 22$ Abs. 2 Landkreisordnung Rheinland-Pfalz & 5 & Nein \\
\hline Saarland & $\begin{array}{l}\S 156 \text { Abs. } 2 \text { Kommunalselbstverwaltungsgesetz } \\
\text { Saarland }\end{array}$ & 4 & Nein \\
\hline Sachsen & $\begin{array}{l}\S 25 \text { Abs. } 2 \text { Landkreisordnung für den Freistaat } \\
\text { Sachsen }\end{array}$ & 5 & Nein \\
\hline Sachsen-Anhalt & $\begin{array}{l}\text { § } 25 \text { Abs. } 3 \text { Landkreisordnung für das Land } \\
\text { Sachsen-Anhalt }\end{array}$ & 4 & Nein \\
\hline Schleswig-Holstein & $\begin{array}{l}\S 8 \mathrm{Nr} .3 \text { Gesetz über die Wahlen in den Gemein- } \\
\text { den und Kreisen in Schleswig-Holstein }\end{array}$ & 2 & Nein \\
\hline Thüringen & $\S 102$ Abs. 3 Thüringer Kommunalordnung & 3 & Nein \\
\hline
\end{tabular}

Tabelle 5: Ländervergleich der Kommunalverfassungen/-wahlgesetze (Kreistage)

Quelle: Eigene Darstellung

Die feingliedrigste Struktur für die Kreistage weist erneut das Land Niedersachsen aus (zehn Größenklassen). Mecklenburg-Vorpommern und Schleswig-Holstein greifen demgegenüber auf ein Größenklassensystem mit lediglich zwei Ebenen zurück. Eine Besonderheit ist im Land Baden-Württemberg zu finden. Als einziges Bundesland nennt es die Größenklassen nicht explizit, sondern beschreibt das Berechnungsverfahren, nach dem sich die Anzahl der Kreistagssitze berechnet ( $\$ 20$ Abs. 2 Landkreisordnung für Baden-Württemberg).

Ein Optionsrecht zur freiwilligen Verkleinerung der Zahl der Kreistagssitze ist in den Kommunalverfassungen/-wahlgesetzen von Brandenburg, Hessen, Niedersachsen und Nordrhein-Westfalen vorgesehen. Baden-Württemberg und Sachsen sind die einzigen Länder, die ein solches Optionsrecht ausschließlich für die Städte-/Gemeindeebene, nicht aber für die Landkreisebene, vorsehen. Wie auch auf Ebene der Städte und Gemeinden verlangt einzig das Land Hessen eine 2/3-Mehrheit zur freiwilligen Verkleinerung der Anzahl der Kreistagssitze.

Aufgrund der uneinheitlichen Untergliederung (insb. bzgl. Anzahl der Größenklassen und Festlegung der Grenzwerte) in den jeweiligen Kommunalverfassungen werden bei den Landkreisen unmittelbare Vergleiche erschwert. Aus diesem Grund wird (analog zur Zusammensetzung der 
Räte in Abschnitt IV.1) eine Vereinheitlichung der Einwohnergrößenklassen vorgenommen (siehe Tab. 6).

\begin{tabular}{|l|c|c|c|c|}
\hline Flächenland & $\begin{array}{c}\text { Bis 100.000 } \\
\text { Einwohner }\end{array}$ & $\begin{array}{c}\mathbf{1 0 0 . 0 0 1 - 2 0 0 . 0 0 0} \\
\text { Einwohner }\end{array}$ & $\begin{array}{c}\mathbf{2 0 0 . 0 0 1 - 3 0 0 . 0 0 0} \\
\text { Einwohner }\end{array}$ & $\begin{array}{c}\text { Ab 300.001 } \\
\text { Einwohnern }\end{array}$ \\
\hline Baden-Württemberg & --- & $36-54$ & $56-64$ & $66-100$ \\
\hline Bayern & $50-60$ & $60-70$ & 70 & 70 \\
\hline Brandenburg & 46 & $50-56$ & 56 & --- \\
\hline Hessen & 51 & $61-71$ & 81 & $87-93$ \\
\hline $\begin{array}{l}\text { Mecklenburg- } \\
\text { Vorpommern }\end{array}$ & 61 & $61-69$ & --- & --- \\
\hline Niedersachsen & 42 & $46-58$ & $62-64$ & $66-70$ \\
\hline Nordrhein-Westfalen & --- & 48 & 54 & $60-72$ \\
\hline Rheinland-Pfalz & $34-42$ & $42-50$ & 50 & --- \\
\hline Saarland & 27 & 33 & 39 & 45 \\
\hline Sachsen & --- & --- & $80-92$ & 98 \\
\hline Sachsen-Anhalt & 42 & $48-54$ & 60 & --- \\
\hline Schleswig-Holstein & --- & 45 & 49 & 49 \\
\hline Thüringen & $40-46$ & $46-50$ & --- & -- \\
\hline Durchschnitt & 45,00 & 51,45 & 60,73 & 71,13 \\
\hline
\end{tabular}

Tabelle 6: Zusammensetzung des Kreistags in den 13 Flächenländern nach vereinheitlichten Größenklassen

Quelle: Eigene Darstellung

Die größten Kreistage haben die Landkreise der Länder Hessen und Sachsen. Die sächsischen Kreistage sind bei gleicher Einwohnerzahl hierbei i. d. R. etwas größer als ihre hessischen Pendants. Ebenfalls als vergleichsweise groß zu kategorisieren sind die Kreistage Mecklenburg-Vorpommerns. Die Landkreise des Saarlandes haben demgegenüber die kleinsten Kreistage. Ebenfalls eher klein sind die Kreistage im Land Schleswig-Holstein. Ähnliche Größenverhältnisse wie in Schleswig-Holstein sind auch für das Land Rheinland-Pfalz festzuhalten. Für Baden-Württemberg ist ein heterogenes Bild festzustellen: Während in den ersten drei Größenklassen mittelgroße Kreistage bestehen, weist Baden-Württemberg in der vierten Größenklasse mit 100 Kreistagssitzen den höchsten Maximalwert aller Länder aus.

Ein eindeutiger Zusammenhang zwischen der Größe des Kreistags und der finanziellen Lage ist für die umlagefinanzierte Kreisebene im Gegensatz zur Stadt-/Gemeindeebene nicht erkennbar. Während sich die hessischen Kommunalfinanzen in einer vergleichsweise schlechten Situation befinden, können die sächsischen Kommunalfinanzen zu den solidesten in Deutschland gezählt werden. Die Finanzen der saarländischen Kommunen sind als eher schlecht zu kategorisieren, während die saarländischen Landkreise gleichzeitig die kleinsten Kreistage aufweisen. ${ }^{11}$

11 Zur Lage der Kommunalfinanzen in den 13 Flächenländern siehe Abschnitt III. 


\section{Zusammenhang zwischen der Größe kommunaler Volksvertretungen und der finanziellen Lage der Kommunen}

\section{Vorgehen und Datengrundlage}

In Abschnitt IV.1 ist ein Indiz für das Vorliegen eines Zusammenhangs zwischen der Größe des Rats und der finanziellen Lage der Städte und Gemeinden identifiziert worden. Auf Grundlage dieser Erkenntnisse kann die These aufgestellt werden, dass Städte/Gemeinden mit großen Räten überdurchschnittlich große Probleme mit den städtischen/gemeindlichen Finanzen haben. Entsprechende Erkenntnisse haben Gilligan/Matsusaka im Hinblick auf erhöhte Ausgaben großer staatlicher Vertretungskörperschaften gewonnen (Gilligan/Matsusaka 2001); (Gilligan/Matsusaka 1995). Hieraus kann abgeleitet werden, dass mit einem höheren Ausgabenniveau ceteris paribus eine schlechtere Haushalts- und Finanzlage einhergeht. ${ }^{12}$

Ein logischer Erklärungsansatz für das potentielle Vorliegen dieses kausalen Zusammenhangs ist, dass aufgrund der großen Anzahl an Ratsmitgliedern auch viele kleine Fraktionen bzw. einzelne fraktionslose Kommunalpolitiker im Rat sitzen. Diese Zersplitterung der Räte kann Haushaltskonsolidierungsmaßnahmen erschweren. ${ }^{13}$ Durch ihre Präsenz in der lokalen Presse können vergleichsweise unbedeutende Splittergruppen immer neue Ausgabewünsche für einzelne Gruppen einbringen, ${ }^{14}$ die von der jeweils begünstigten Wählergruppe goutiert werden - wohlwissend, dass die Splittergruppen selbst für die negativen Folgen ihrer Ausgabenwünsche (insb. höhere Verschuldung) nicht zur Rechenschaft gezogen werden. Für die schlechte finanzielle Lage werden vielmehr die großen Fraktionen verantwortlich gemacht. Die großen Fraktionen sehen sich damit einem Spagat zwischen notwendiger Haushaltskonsolidierung einerseits und der nächsten Kommunalwahl andererseits gegenüber. In Anbetracht der bedenklichen Finanzlage in den Städten/ Gemeinden der Länder Hessen, Nordrhein-Westfalen, Rheinland-Pfalz und Saarland scheint dieser Spagat nur in wenigen Städte/Gemeinden mit großem Rat zu gelingen.

Ferner haben Auriol/Gary-Bobo herausgefunden, dass große Volksvertretungen den Grad an Bürokratie steigern, Unternehmertum einschränken und Korruption erleichtern (Auriol/Gary-Bobo 2008). Bezüglich dieser Faktoren ist davon auszugehen, dass sie einen negativen Einfluss auf die Kommunalfinanzsituation haben (z. B. höhere Verwaltungsausgaben, geringere Gewerbesteuereinnahmen).

12 Einschränkend ist darauf hinzuweisen, dass hohe Ausgaben nicht notwendigerweise eine schlechte Haushaltsund Finanzsituation zur Folge haben müssen. Sofern hohe Ausgaben durch entsprechend hohe Einnahmen gedeckt sind, ist der Haushaltsausgleich durch ein hohes Ausgabenniveau nicht gefährdet.

13 So hängen z. B. Effizienz und Effektivität einer Volksvertretung nach Muzzio/Tompkins von deren Größe ab. Große, zersplitterte Volksvertretungen führen demnach u. a. zu häufigeren Konflikten (Muzzio/Tompkins 1989, S. 94). Gegenstand dieser Konflikte können beispielsweise Fragen der Haushaltskonsolidierung sein. Auch der Bund der Steuerzahler Hessen sieht in der Zersplitterung der Volksvertretungen eines der Hauptprobleme für die Effizienz der Arbeit der Volksvertreter (Bund der Steuerzahler Hessen e. V. 2010 a, S. 11). Einschränkend ist anzumerken, dass die Fragmentierung der Volksvertretungen neben der Größe u. a. auch von anderen Regelungen im Kommunalwahlrecht abhängen kann. Bedingt durch den unverhältnismäßig hohen Erhebungsaufwand einer Online-Recherche bei knapp 3.250 Kommunen (ab 5.000 Einwohnern), ist die Variable „Fragmentierung der Volksvertretung“ jedoch nicht in die Analyse einbezogen worden.

14 Zur Theorie, dass öffentliche Ausgaben primär einzelnen, kleineren Gruppen Nutzen stiften siehe Tullock 1959; Buchanan/Tullock 1962. 
Der Frage nach dem Vorliegen eines Kausalzusammenhangs zwischen der Größe der kommunalen Volksvertretung und der kommunalen Haushalts- und Finanzlage soll im vorliegenden Abschnitt im Rahmen einer Regressionsanalyse untersucht werden. Als Indikatoren für die Operationalisierung der Haushalts- und Finanzlage der Kommunen kommen das ordentliche Ergebnis (Kernhaushalt) je Einwohner sowie der Stand der Kassenkredite je Einwohner ${ }^{15}$ zur Anwendung. ${ }^{16}$

Das ordentliche Ergebnis ${ }^{17}$ ist die Summe aus Primärergebnis und Finanzergebnis. Das Primärergebnis bestimmt sich aus dem Saldo der laufenden Einnahmen und Ausgaben (Bertelsmann Stiftung 2011, S. 42). Das Finanzergebnis berechnet sich über den Saldo aus Zinseinnahmen/ Dividenden und Zinsausgaben (Bertelsmann Stiftung 2011, S. 42). Das ordentliche Ergebnis ist damit eine stark aggregierte Kennziffer zur Beurteilung der finanziellen Situation der Kommune im betreffenden Haushaltsjahr. Kassenkredite sind kurzfristige Kredite zur Liquiditätssicherung (Burth u. a. 2012, S. 218). Auf die Betrachtung der übrigen Schuldenarten ist verzichtet worden, da z. B. Investitionskredite nicht per se etwas Schlechtes darstellen. ${ }^{18}$ Hohe Kassenkreditstände sind demgegenüber ein klassisches Krisenphänomen (Gnädinger 2011, S. 72 ff.); (Junkernheinrich/Micosatt 2008, S. 17); (Heinemann u. a. 2009, S. 17). Folglich kann von hohen Kassenkreditbeständen auf eine schlechte finanzielle Lage geschlossen werden.

15 Es ist darauf hinzuweisen, dass Kassenkreditbestände in ihrer Höhe z. T. auch „Altlasten“ früherer Jahre darstellen, die nicht bzw. nur teilweise von den heutigen Kommunalpolitikern verursacht worden sind. Gleichwohl kann davon ausgegangen werden, dass eine kommunale Volksvertretung hinsichtlich ihrer Größe (unabhängige Variable) im Zeitablauf weitestgehend konstant bleibt bzw. nur geringfügigen Änderungen unterliegt (z. B. Einwohnergrenze wird über-/unterschritten, Änderung der Kommunalverfassung). Es kann damit die Annahme getroffen werden, dass die unabhängige Variable zum Zeitpunkt des Entstehens der Kassenkreditbestände (näherungsweise) die gleichen Werte angenommen hat wie heute. Die heutige Volksvertretungsgröße stellt demnach ein Spiegelbild der Größe der Vergangenheit dar und ist folglich in ihrer Höhe ursächlich für das Volumen heutiger Kassenkreditbestände.

Ferner sind die heutigen Kommunalpolitiker dahingehend (mit-)verantwortlich für die Höhe der heutigen Kassenkreditbestände, als dass sie den Höchstbetrag an Kassenkrediten jedes Jahr in der Haushaltssatzung festlegen. Aufgrund ihrer sehr kurzen Laufzeit werden Kassenkredite (im Gegensatz zu den langfristigen Investitionskrediten) rollierend aufgenommen.

16 Im vorliegenden Beitrag ist auf einen monokausalen Ansatz zurückgegriffen worden, um im Untersuchungsmodell zu betonen, dass die Größe der Volksvertretungen, d. h. die unabhängige Variable, im Fokus der Untersuchung steht. Die Kommunalfinanzsituation ist demgegenüber über zwei (abhängige) Variablen breiter operationalisiert worden.

17 Beim ordentlichen Ergebnis handelt es sich im vorliegenden Fall um eine kamerale Kenngröße. Das doppische Äquivalent (ordentliches Jahresergebnis der Kernverwaltung) wäre grundsätzlich aufgrund der Ressourcenverbrauchs- bzw. Ressourcenaufkommensorientierung im Sinne des Grundsatzes der Generationengerechtigkeit der geeignetere Ansatz zur Beurteilung der aktuellen Haushaltslage. Diese Kennzahl steht gleichwohl finanzstatistisch nicht zur Verfügung, weshalb im vorliegenden Beitrag auf das kamerale ordentliche Ergebnis zurückgegriffen werden muss. Selbiges gilt analog für das ordentliche Jahresergebnis des Konzerns Kommune (Gesamt-/Konzernabschluss) sowie die Eigenkapitalquote (Kernverwaltung sowie Konzern Kommune). Die Betrachtung des gesamten Konzerns Kommune (Kernverwaltung und Auslagerungen) wäre grundsätzlich gegenüber der isolierten Betrachtung der Kernverwaltung zu bevorzugen, da Kommunen oft große Teile ihrer Aufgabenerfüllung in Auslagerungen (z. B. Eigenbetriebe) wahrnehmen. Zur finanzwirtschaftlichen Bedeutung von kommunalen Auslagerungen siehe Junkernheinrich/Micosatt 2008, S. $92 \mathrm{ff}$.

18 Hintergrund ist, dass Investitionskredite i. d. R. für die Schaffung von (materiellen) Vermögensgegenständen aufgenommen werden. Demzufolge steht diesen Krediten ein materieller Vermögenswert gegenüber. Bei Kassenkrediten ist dies nicht der Fall, da sie v. a. für laufende Ausgaben (z. B. Personalausgaben) aufgenommen werden (Junkernheinrich/Micosatt 2008, S. 20). 
Als Hypothesen können damit formuliert werden:

Hypothese 1: $\quad$ Die Anzahl der Mitglieder kommunaler Volksvertretungen steht in einem negativen Kausalzusammenhang zum ordentlichen Ergebnis je Einwohner.

Hypothese 2: $\quad$ Die Anzahl der Mitglieder kommunaler Volksvertretungen steht in einem positiven Kausalzusammenhang zum Kassenkreditbestand je Einwohner.

Die Einnahme-, Ausgabe- und Aufgabenstruktur der Kommunen unterscheidet sich indes abhängig vom Kommunaltyp (kreisangehörige Städte/Gemeinden, kreisfreie Städte, Landkreise) ${ }^{19}$ Es ist erscheint demnach angebracht, den Kommunaltyp als Kontrollvariable einzuführen und die Analyse für jeden Kommunaltyp differenziert vorzunehmen.

Für dem Kommunaltyp „kreisangehörige Städte/Gemeinden“ ergeben sich damit folgende (Sub-)Hypothesen:

Hypothese 1 a: $\quad$ Die Anzahl der Ratsmitglieder kreisangehöriger Städte/Gemeinden steht in einem negativen Kausalzusammenhang zum ordentlichen Ergebnis je Einwohner.

Hypothese 2a: Die Anzahl der Ratsmitglieder kreisangehöriger Städte/Gemeinden steht in einem positiven Kausalzusammenhang zum Kassenkreditbestand je Einwohner.

Analog hierzu lauten die (Sub-)Hypothesen für den Kommunaltyp „kreisfreie Städte“:

Hypothese 1 b: $\quad$ Die Anzahl der Ratsmitglieder kreisfreier Städte steht in einem negativen Kausalzusammenhang zum ordentlichen Ergebnis je Einwohner.

Hypothese 2 b: $\quad$ Die Anzahl der Ratsmitglieder kreisfreier Städte steht in einem positiven Kausalzusammenhang zum Kassenkreditbestand je Einwohner.

Indizien für das Vorliegen eines Kausalzusammenhangs sind in Abschnitt IV primär für die Städte und Gemeinden identifiziert worden. Der Vollständigkeit halber soll die Untersuchung auch für Landkreise vorgenommen werden, um auch hier einen Nachweis für das Vorliegen bzw. NichtVorliegen eines Kausalzusammenhangs (sowie dessen Richtung und Stärke) liefern zu können. Die (Sub-)Hypothesen sind in Analogie zu den Hypothesen der Städte und Gemeinden formuliert worden, da für die Kreise keine spezifischen Erkenntnisse über eine Andersartigkeit des Zusammenhangs vorliegen.

Hypothese 1 c: $\quad$ Die Anzahl der Kreistagsmitglieder steht in einem negativen Kausalzusammenhang zum ordentlichen Ergebnis je Einwohner.

19 So nehmen beispielsweise kreisfreie Städte sowohl die Aufgaben einer kreisangehörigen Stadt/Gemeinde als auch die Aufgaben eines Landkreises wahr. Bedingt durch verschiedenartige Aufgabenstrukturen unterscheidet sich die Ausgabestruktur zwischen den Kommunaltypen. Die Einnahmestruktur der Landkreise weist die Besonderheit auf, dass diese sich als Umlageverbände primär über die von den kreisangehörigen Städten/Gemeinden zu entrichtende Kreisumlage finanzieren. Voluminöse eigene Steuereinnahmequellen (wie z. B. die Realsteuern bei den kreisfreien und kreisangehörigen Städten und Gemeinden) weisen Landkreise i. d. R. nicht auf. 
Der Effekt der Größe kommunaler Volksvertretungen auf die Kommunalfinanzsituation

Hypothese 2 c:

Die Anzahl der Kreistagsmitglieder steht in einem positiven Kausalzusammenhang zum Kassenkreditbestand je Einwohner.

Die Hypothesen lassen sich mithin zu folgendem Untersuchungsmodell verdichten:

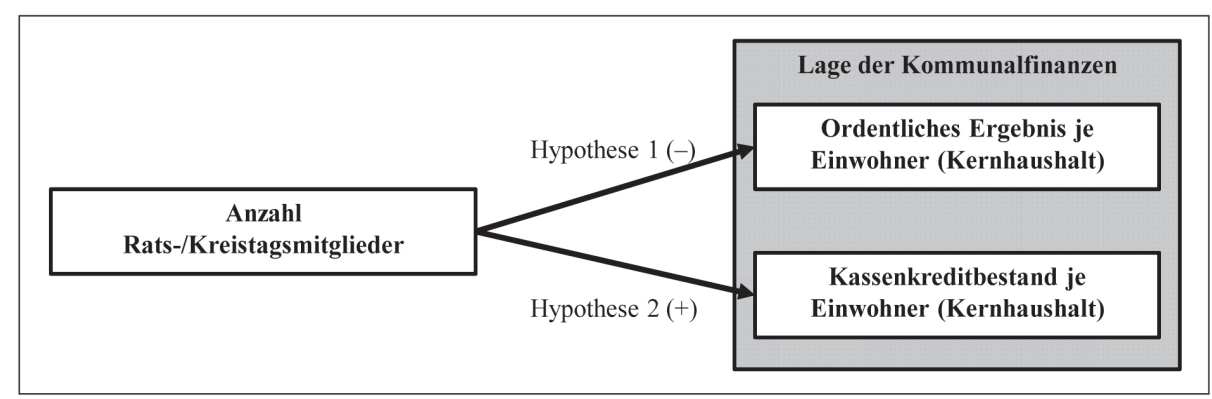

Abbildung 1: Untersuchungsmodell

Quelle: Eigene Darstellung

Die Datengrundlage zum ordentlichen Ergebnis sowie zu den Kassenkreditbeständen sind dem Autor freundlicherweise von der Bertelsmann Stiftung (Programm LebensWerte Kommune) für alle Landkreisverwaltungen sowie alle Städte und Gemeinden ab 5.000 Einwohnern für die Jahre 2006 bis 2009 zur Verfügung gestellt worden. ${ }^{20}$ Gleiches gilt für die Einwohnerzahlen der Kommunen, die ebenfalls von der Bertelsmann Stiftung bereitgestellt und vom Autor auf Basis der einschlägigen Rechtsregelungen in die Anzahl der Mandatsträger umgerechnet worden sind. ${ }^{21}$

Um sicherzustellen, dass das ordentliche Ergebnis nicht durch außerordentliche Effekte/Ereignisse beeinflusst worden ist, ist das ordentliche Ergebnis über eine Mehrjahresbetrachtung als arithmetisches Mittel der Jahre 2006 bis 2008 berechnet worden. ${ }^{22}$ Für den Bestand an Kassenkrediten sind die Daten aus dem Jahr 2008 herangezogen worden. ${ }^{23}$ Im Falle der Kassenkredite ist kein Mehrjahresdurchschnitt bestimmt worden, da es sich bei den Kassenkrediten - im Gegensatz zum ordentlichen Ergebnis - um eine Bestandsgröße und nicht um eine Stromgröße handelt.

Sowohl beim ordentlichen Ergebnis als auch bei den Kassenkrediten ist das Jahr 2009 nicht berücksichtigt worden. Grund hierfür ist erstens, dass die Daten aus diesem Jahr erheblich durch die

20 Aufgrund dessen, dass lediglich die Daten der Kommunen ab 5.000 Einwohnern zur Verfügung stehen, können die Ergebnisse nicht bzw. nur begrenzt auf Kommunen unter 5.000 Einwohner übertragen werden.

21 Zur Umrechnung sind die Einwohnerzahlen für das Jahr 2008 verwendet worden, da auch bei den übrigen Kennzahlen das Jahr 2009 nicht berücksichtigt worden ist. Bedingt durch das Umrechnungsverfahren aus den Einwohnerzahlen können sich in den Fällen der Kommunen, deren Einwohnerzahl seit der vorangegangenen Kommunalwahl eine einschlägige, kommunalrechtliche Einwohnergrenze unter- oder überschritten hat, Abweichungen zur tatsächlichen Größe der Volksvertretung ergeben. Die Verzerrungen sind jedoch vermutlich von geringfügigem Charakter und sollten die Regressionsanalyse nicht wesentlich beeinflussen.

22 Aufgrund von Lücken im Datensatz können nicht alle Städte und Gemeinden ab 5.000 Einwohnern von der vorliegenden Analyse abgedeckt werden. Bei 32 von 2.951 Städten/Gemeinden (1,08 \%) stehen nur aus zwei oder weniger Jahren Daten zur Verfügung, weshalb selbige aus der Untersuchung eliminiert worden sind. Bei den Landkreisen sind keine Lücken im Datensatz vorhanden.

23 Bei 13 von 2.951 Städten und Gemeinden (0,44 \%) sind keine Kassenkreditbestände für das Jahr 2008 verfügbar. Die entsprechenden Datensätze sind daher aus der Analyse eliminiert worden. 
Finanz- und Wirtschaftskrise verzerrt worden sind. ${ }^{24}$ Zweitens handelt es sich bei den 2009 erDaten um kassenstatische Daten, die aufgrund ihres vorläufigen Charakters weniger valide sind als die Rechnungsergebnisdaten aus den Jahren 2006 bis 2008. In Abwägung von Aktualität einerseits und Validität andererseits ist bezüglich der Kassenkreditbestände eine Entscheidung zugunsten der Validität der Daten gefallen, zumal die Aktualität durch ein Jahr ältere Daten nicht wesentlich negativ beeinflusst wird. Drittens sind die Daten aus dem Jahr 2009 - im Gegensatz zu den Daten aus den Jahren 2006 bis 2008 - durch größere Datenlücken geprägt. ${ }^{25}$

In Ländern mit Optionsrecht zur freiwilligen Verkleinerung der Anzahl der Rats-/Kreistagsmitglieder ist eine pauschale, prozentuale Verringerung der Mitgliederzahlen vorgenommen worden. Für das Land Hessen beläuft sich die Verringerung in Analogie zu den Ergebnissen einer Studie des Bundes der Steuerzahler Hessen auf 4,2\% (Bund der Steuerzahler Hessen e. V. 2010 a, S. 33). Für die übrigen Länder mit Optionsrecht liegen dem Autor keine spezifischen Studien vor. Die Anzahl der Sitze ist in diesen Ländern um 6\% verringert worden. Hintergrund des etwas höheren Prozentwertes ist, dass Hessen mit einer 2/3-Mehrheit höhere Hürden zur Verkleinerung auferlegt als die übrigen Länder mit Optionsrecht. Es wird daher davon ausgegangen, dass sich in den Ländern außer Hessen mehr Kommunen für eine Verringerung der Anzahl der Sitze entschieden haben.

\section{Ergebnisse der Regressionsanalyse}

Zum Zweck des Testens der Hypothesen kommt eine lineare Regressionsanalyse zur Anwendung. Die betrachteten Variablen (Kassenkreditbestand je Einwohner, ordentliches Ergebnis je Einwohner, Anzahl Sitze) sind metrisch skaliert.

Bei Betrachtung einzelner Datenelemente fällt jedoch auf, dass es einzelne Kommunen (Ausreißer) gibt, die ausgesprochen hohe Werte bei den Kenngrößen zur Finanzlage ausweisen (wie z. B. die Gemeinde Grünwald in Bayern, deren ordentliches Ergebnis im arithmetischen Mittel der Jahre 2006 bis 2008 bei +6.356,05 Euro je Einwohner liegt - bei einem arithmetischen Mittel von +29,29 Euro je Einwohner für den Durchschnitt sämtlicher Städte und Gemeinden). Datensätze dieser Art stellen zwar keine Messfehler, sondern echte Werte dar, gleichwohl haben sie zur Folge, dass die Regression auf Basis der Methode der kleinsten Quadrate stark verzerrt werden kann, wodurch die Datenstruktur von der Regressionsgeraden nicht mehr adäquat abgebildet wird (Fahrmeier/Kneib/Lang 2009, S. 173 ff.); (Pflaumer/Heine/Hartung 2005, S. 162); (Hawkins 1980). Um Ausreißer vor Durchführung der Regressionsanalyse zu identifizieren und aus der Analyse zu eliminieren, werden die sog. Externally Studentized Residuals verwendet. Hierbei wird für sämtliche Beobachtungen die Regression ohne die jeweilige Beobachtung errechnet. Die standardisierte Differenz hinsichtlich der abhängigen Variablen für die jeweilige Beobachtung wird als Externally Studentized Residual bezeichnet. Die Externally Studentized Residuals sind hierbei t-verteilt. Einen allgemein anerkannten, kritischen Wert von Externally Studentized Re-

24 Zu den Auswirkungen der Finanz- und Wirtschaftskrise auf die Kommunalfinanzen siehe Abschnitt III.

25 So liegen z. B. für das Jahr 2009 bei 458 Städten und Gemeinden ab 5.000 Einwohnern (15,52\%) keine Daten für das ordentliche Ergebnis vor. Für die Länder Hessen und Saarland fehlen die entsprechenden Daten des ordentlichen Ergebnisses für 2009 komplett - beim Land Brandenburg liegt die Datenlücke bei 66,27\%. 
siduals, ab dem von einem Ausreißer gesprochen wird, existiert gleichwohl nicht. In Analogie zu Cohen u. a. werden für den vorliegenden Beitrag Werte über $+2,00$ sowie Werte unter $-2,00$ als Ausreißer klassifiziert (Cohen u. a. 2003, S. 401 ff.). Tabelle 7 enthält die Ergebnisse der Regressionsanalyse unter Ausschluss derjenigen Daten, die auf Basis der Externally Studentized Residuals als Ausreißer klassifiziert worden sind. ${ }^{26}$

\begin{tabular}{|l|c|c|c|c|c|c|}
\hline Flächenland & $\begin{array}{c}\text { Regres- } \\
\text { sionskoef- } \\
\text { fizient }\end{array}$ & $\begin{array}{c}\text { Kon- } \\
\text { stante }\end{array}$ & $\begin{array}{c}\text { Be- } \\
\text { stimmt- } \\
\text { heitsmaß } \\
\text { (R2) }\end{array}$ & $\mathbf{p}$ & $\begin{array}{c}\text { N } \\
\text { (ohne } \\
\text { Ausrei- } \\
\text { Ber) }\end{array}$ & $\begin{array}{c}\text { Anzahl } \\
\text { elimi- } \\
\text { nierte } \\
\text { Ausrei- } \\
\text { Ber }\end{array}$ \\
\hline $\begin{array}{l}\text { Hypothese 1 a: } \\
\text { Anzahl Sitze } \rightarrow \text { Kassenkredite (+) }\end{array}$ & $5,052^{* * *}$ & $-79,802$ & 0,082 & 0,000 & 2.722 & 113 \\
\hline $\begin{array}{l}\text { Hypothese 2 a: } \\
\text { Anzahl Sitze } \rightarrow \text { ordentl. Ergebnis } \\
(-)\end{array}$ & $-2,358^{* * *}$ & 113,658 & 0,026 & 0,000 & 2.765 & 51 \\
\hline $\begin{array}{l}\text { Hypothese 1 b: } \\
\text { Anzahl Sitze } \rightarrow \text { Kassenkredite (+) }\end{array}$ & $25,210^{* * *}$ & $-714,787$ & 0,123 & 0,000 & 98 & 5 \\
\hline $\begin{array}{l}\text { Hypothese 2 b: } \\
\text { Anzahl Sitze } \rightarrow \text { ordentl. Ergebnis } \\
(-)\end{array}$ & $-0,825$ & 43,815 & 0,003 & 0,576 & 95 & 8 \\
\hline $\begin{array}{l}\text { Hypothese 1 c: } \\
\text { Anzahl Sitze } \rightarrow \text { Kassenkredite (+) }\end{array}$ & $-1,387^{*}$ & 155,263 & 0,016 & 0,035 & 286 & 15 \\
\hline $\begin{array}{l}\text { Hypothese 2 c: } \\
\text { Anzahl Sitze } \rightarrow \text { ordentl. Ergebnis } \\
(-)\end{array}$ & $0,347^{*}$ & $-13,871$ & 0,018 & 0,026 & 280 & 21 \\
\hline
\end{tabular}

Tabelle 7: Ergebnisse der Regressionsanalyse (nach Eliminierung der Ausreißer mittels Externally Studentized Residuals) *** = Signifikanzniveau von 0,001 .

Quelle: Eigene Berechnung

$* * *=$ Signifikanzniveau von 0,001
$* *=$ Signifikanzniveau von 0,01
$*=$ Signifikanzniveau von 0,05

$(+) /(-)=$ Hypothese eines positiven/negativen Kausalzusammenhangs

Im Ergebnis ist für fünf der sechs Hypothesen eine signifikante bis hoch signifikante Kausalbeziehung identifiziert worden. ${ }^{27}$ Die Hypothesen 1 a und 2 a (kreisangehörige Städte/Gemeinden) sind hierbei bestätigt worden. Es wird jeweils ein sehr hohes Signifikanzniveau erreicht. Ein ebenfalls sehr hohes Signifikanzniveau wird für Hypothese $1 \mathrm{~b}$ (Kassenkredite; kreisfreie Städte) nachgewiesen. Nicht signifikant ist demgegenüber Hypothese $2 \mathrm{~b}$ (ordentliches Ergebnis; kreis-

26 Prozentual sind zwischen 1,84\% und 7,77\% der Kommunen als Ausreißer klassifiziert und als solche eliminiert worden. Aufgrund der vergleichsweise geringen Prozentwerte fließt die Mehrzahl der Beobachtungen weiterhin in die Regressionsanalyse ein.

27 Es sei darauf hingewiesen, dass nicht ausgeschlossen werden kann, dass es sich bei den identifizierten Beziehungen jeweils um Scheinkorrelationen bzw. von einer dritten Variablen (z. B. Einwohnerzahl) determinierte Korrelationen handelt. 
freie Städte). Für die Hypothesen $1 \mathrm{c}$ und $2 \mathrm{c}$ (Landkreise) haben sich auf einem Niveau von 0,05 signifikante Kausalbeziehungen mit umgekehrtem Vorzeichen ergeben.

Aus den Ergebnissen kann geschlussfolgert werden, dass im Fall von kreisangehörigen und kreisfreien Städten und Gemeinden eine Verkleinerung der Volksvertretung zu einer Verbesserung der Haushalts- und Finanzlage führt, wenngleich bezüglich der kreisfreien Städte einzuschränken ist, dass dies lediglich für die Kassenkreditbestände gilt. Im Hinblick auf die Landkreise zeigen die empirischen Ergebnisse, dass eine Vergrößerung der Kreistage einen positiven Effekt auf die Lage der Kommunalfinanzen hat.

Zwar sind für fünf Hypothesen signifikante Zusammenhänge entdeckt worden, gleichwohl unterscheidet sich die Stärke des Effekts zwischen den einzelnen Hypothesen. In Anlehnung an die Interpretationsempfehlungen für Bestimmtheitsmaße nach Cohen (Cohen 1988, S. 531 ff.) und Rubin (Rubin 2009, S. 138 ff.) ist ein mittelstarker Zusammenhang für die kreisangehörigen Städte und Gemeinden bzgl. Hypothese $1 \mathrm{a}\left(\mathrm{R}^{2}=0,082\right)$ und die kreisfreien Städte bzgl. Hypothese $1 \mathrm{~b}$ $\left(\mathrm{R}^{2}=0,123\right)$ identifiziert worden. Für die Städte und Gemeinden gilt demnach, dass Verringerungen der Ratssitze zu einer mittelstarken Verminderung des Kassenkreditbestandes führen. Ein schwacher Zusammenhang ist für die Hypothesen 2 a $\left(\mathrm{R}^{2}=0,026\right), 2 \mathrm{c}\left(\mathrm{R}^{2}=0,018\right)$ und $1 \mathrm{c}$ $\left(\mathrm{R}^{2}=0,016\right)$ festzuhalten. Im Falle der Landkreise hat demnach eine Erhöhung der Anzahl der Kreistagssitze einen schwachen positiven Effekt auf das ordentliche Ergebnis sowie einen schwachen negativen Effekt auf den Kassenkreditbestand. Bei kreisangehörigen Städten und Gemeinden haben größere Räte einen schwachen negativen Einfluss auf die Höhe des ordentlichen Ergebnisses.

Die beschriebenen Ergebnisse (unterschiedliches Vorzeichen für Räte und Kreistage) überraschen auf den ersten Blick. Gleichwohl liefert die bisherige Forschung zur optimalen Größe von Volksvertretungen einen geeigneten Ansatzpunkt für die Erklärung dieses Phänomens. ${ }^{28}$ Gegeben der Annahme, dass aus finanzwirtschaftlicher Sicht eine optimale Größe für eine kommunale Volksvertretung existiert, so wäre es darüber hinaus plausibel davon auszugehen, dass es aufgrund der unterschiedlichen Ausgaben-, Einnahmen- und Aufgabenstruktur Unterschiede in der optimalen, finanzwirtschaftlichen Größe von Räten bzw. Kreistagen gibt. Aufbauend auf diesen Überlegungen lassen sich die Beobachtungen insofern erklären, als dass die Räte der Städte und Gemeinden derzeit größer sind als die optimale Größe, während die Kreistage unterhalb des optimalen Niveaus liegen. Eine Vergrößerung der Kreistage hat demnach einen positiven Effekt auf die Kommunalfinanzen während der Zusammenhang bei den Räten das jeweils umgekehrte Vorzeichen hat. Aus diesen Überlegungen lässt sich schlussfolgern, dass die Richtung des Effekts einer Änderung in der Größe einer Volksvertretung davon abhängt, ob die jeweilige Volksvertretung ober- oder unterhalb des Optimums liegt.

Da der kausale Effekt bei den (kreisangehörigen und kreisfreien) Städten und Gemeinden stärker ist als bei den Landkreisen, ist zu vermuten, dass sich die Städten und Gemeinden vergleichsweise weiter vom Optimum entfernt befinden als die Landkreise. Das Konsolidierungspotential durch Veränderungen der Größe der Volksvertretungen ist demnach bei den Städten und Gemeinden größer als bei den Landkreisen. 
Abbildung 2 verdeutlicht den oben beschriebenen Zusammenhang zwischen Größe einer Volksvertretung und kommunaler Finanzlage skizzenhaft für die Städte/Gemeinden sowie die Landkreise.

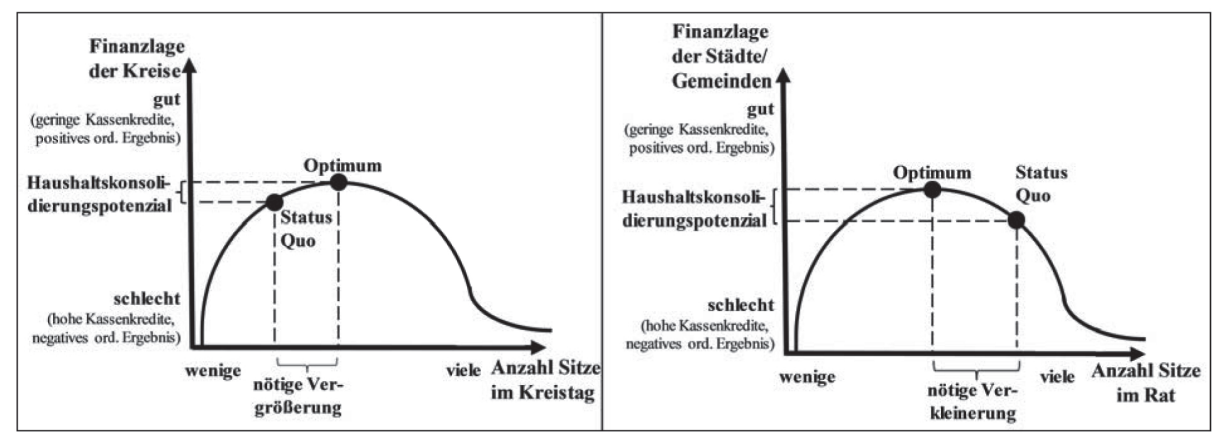

Abbildung 2: Skizzierung der Haushaltskonsolidierungspotentiale kommunaler Volksvertretung in Bezug auf die optimale Volksvertretungsgröß $e^{29}$

Quelle: Eigene Darstellung

Geht man davon aus, dass der in Abbildung 2 aufgezeigte Funktionsverlauf näherungsweise zutrifft, so hat die in Regressionsgerade den Charakter einer Tangente am Status-Quo-Punkt. Die Regressionsgerade zeigt folglich an, um wie stark sich die Finanzlage einer Kommune (Kassenkreditbestand, ordentliches Ergebnis) durch eine infinitesimale Erhöhung/Senkung der Volksvertretungsgröße ändert. So würde sich der Kassenkreditbestand einer (fiktiven) deutschen kreisangehörigen Stadt/Gemeinde laut Regressionsgerade um rund 5,05 Euro je Einwohner verringern, wenn der Rat um einen Sitz verkleinert wird. Gleichzeitig würde eine Verbesserung von ca. 2,36 Euro je Einwohner im ordentlichen Ergebnis realisiert werden. Im Falle der kreisfreien Städte ist durch eine Verkleinerung des Rates um einen Sitz eine Verringerung des Kassenkreditbestandes um etwa 25,21 Euro je Einwohner zu erwarten. Bei den Kreisen ließe sich durch eine Vergrößerung des Kreistages um einen Sitz eine Verbesserung von knapp 1,40 Euro je Einwohner beim Kassenkreditbestand und rund 0,35 Euro je Einwohner beim ordentlichen Ergebnis erzielen.

\section{Schlussfolgerungen und Fazit}

Zusammenfassend ist festzuhalten, dass Einsparpotentiale - entgegen der Vermutung des Bundes der Steuerzahler Hessen (Bund der Steuerzahler Hessen e. V. 2010 a) - nicht notwendigerweise nur durch eine Verkleinerung der Volksvertretungen realisiert werden können. Vielmehr ist die

29 Auf eine separate Grafik für die kreisfreien Städte ist an dieser Stelle verzichtet worden, um die Abbildung nicht durch ein drittes Diagramm zu überladen. Die kreisfreien Städte sind daher im Diagramm mit den kreisangehörigen Städten und Gemeinden zusammengefasst worden, zumal die Wirkungsrichtung bei beiden Kommunaltypen gleich ist. Ungeachtet dessen ist einschränkend anzumerken, dass die Abbildung im Falle der kreisfreien Städte lediglich für die Kassenkreditbestände, nicht jedoch das ordentliche Ergebnis gilt, da letzteres nicht signifikant ist. 
Frage „Verkleinerung vs. Vergrößerung“ abhängig davon, ob die aktuelle Volksvertretungsgröße rechts oder links des Optimums liegt.

Die im vorliegenden Beitrag abstrakt beschriebene, finanzwirtschaftliche Optimum hinsichtlich der Größe einer kommunalen Volksvertretung, konnte im vorliegenden Beitrag aufgrund des gewählten Untersuchungsansatzes gleichwohl nicht klar bestimmt werden. Insofern ist das Konzept eines finanzwirtschaftlichen Optimums als These (Proposition) zu verstehen. Die These sollte im weiteren Verlauf des Forschungsprozesses von nachfolgenden Forschern kritisch hinterfragt und im Fall der Bestätigung der These konkret bestimmt werden. Gleichzeitig sollte im günstigen Fall anvisiert werden, das bestehende Haushaltskonsolidierungspotenzial zu quantifizieren. Ferner ist denkbar, dass das Optimum bundeslandabhängig ist, zumal aufgrund landesrechtlicher Gegebenheiten Unterschiede in der kommunalen Einnahme-, Ausgabe- und Aufgabenstruktur bestehen. Es erscheint daher angebracht, die Bundesland-Zugehörigkeit als Kontrollvariable einzuführen. ${ }^{30}$ Dies würde auch dem Umstand Rechnung tragen, dass Kassenkreditbestände, z. B. aufgrund unterschiedlich restriktiver Kassenkreditschuldenbremsen (Gnädinger 2011), in den einzelnen Ländern unterschiedliche Niveaus erreichen.

In der vorliegenden Analyse sind Ausreißer eliminiert worden. Bei den ausgeschlossenen Ausreißern handelt es sich gleichwohl um nicht um Messfehler, sondern um reale Werte. Die Ausreißer stellen damit ihrerseits interessante Untersuchungsobjekte dar, die einer gesonderten Ausreißeranalyse unterzogen werden sollten.

Abschließend stellt sich die Frage, wie eine potentielle Verkleinerung/Vergrößerung der kommunalen Volksvertretungen in der Praxis umgesetzt werden kann. Eine erste Möglichkeit stellt die Etablierung von Vergrößerungswahlrechten (für Kreise) bzw. Verkleinerungswahlrechten (für Städte/Gemeinden) in den Kommunalverfassungen der Länder dar. Um freiwillige Änderungen zu erleichtern, sollte lediglich ein Mehrheitsbeschluss, d. h. keine 2/3-Mehrheit, erforderlich sein. Zweitens kann der Landtag durch Änderung der Kommunalverfassung auf eine (erzwungene) Verkleinerung/Vergrößerung der Mandatszahlen hinwirken. Drittens führen Gemeindefusionen indirekt zu einer Verkleinerung der Volksvertretungen, da mit einer Verdopplung der Einwohnerzahl i. d. R. keine Verdopplung der Ratsmitgliederzahl einhergeht. Das Land kann über sog. Hochzeitsprämien Anreize zu Gemeindefusionen setzen. Eine entsprechende Wirkung entfalten ferner auch Gebietsreformen. Gleichwohl ist zu bedenken, dass Gebietsreformen bzw. Gemeindefusionen nicht nur Auswirkungen auf die Größe der kommunalen Volksvertretung haben. So sind z. B. durch eine Gemeindefusion Einsparungen im Verwaltungsapparat realisierbar, die größer sein können als die Haushaltskonsolidierungspotentiale durch die Veränderung der Anzahl der Mandatsträger.

30 Einschränkend ist jedoch darauf hinzuweisen, dass aufgrund niedriger Fallzahlen bei den kreisfreien Städten und Landkreisen in einigen Ländern (z. B. drei kreisfreie Städte in Sachsen, sechs Landkreise im Saarland) fallzahlbedingte Probleme bei der Durchführung der landesspezifischen Regression auftreten können. 
Der Effekt der Größe kommunaler Volksvertretungen auf die Kommunalfinanzsituation

\section{Abstract}

Andreas Burth; The effect of the size of local councils on the situation of local finances

\section{Budget consolidation; County council; Local finances; Municipal council}

Due to the critical situation of local finances, budget consolidation is crucial in many municipalities and counties. The Taxpayer Association of Hesse therefore proposes to reduce the size of local councils. The article at hand descriptively evaluates the situation of local finances as well as the size of local councils, and analyses the savings potential of decreasing the local councils' size. As a result, municipal councils can exploit consolidation potentials by reducing their size, whereas county councils can realize savings potentials by enlargements.

\section{Literaturverzeichnis}

Anton, Stefan/Diemert, Dörte (2009), Gemeindefinanzbericht 2009 - Kurzfassung, Kommunalfinanzen in freien Fall?, in: der städtetag, Heft 5/2009, S. 5-9.

Anton, Stefan/Diemert, Dörte (2010), Gemeindefinanzbericht 2010 - Kurzfassung, Kommunale Finanzen: Keine Licht am Ende des Tunnels!, in: der städtetag, Heft 5/2010, S. 5-9.

Auriol, Emmanuelle/Gary-Bobo, Robert J. (2008), On the Optimal Number of Representatives, IDEI Working Papers 86 , Toulouse.

Buchanan, James M./Tullock, Gordon (1962), The Calculus of Consent: Logical Foundations of Constitutional Democracy, Ann Arbor.

Budäus, Dietrich/Hilgers, Dennis (2010 a), Eigenkapital/Eigenkapitalquote - ein vernachlässigbarer Faktor?, in: der städtetag, Heft 4/2010, S. 22-26.

Budäus, Dietrich/Hilgers, Dennis (2010 b), Gesamtabschluss von Gebietskörperschaften: Aktuelle Situation und ausgewählte Problemfelder unter besonderer Berücksichtigung der kommunalen Ebene, in: Zeitschrift für öffentliche und gemeinwirtschaftliche Unternehmen, Heft 4/2010, S. 73-95.

Bund der Steuerzahler Hessen e. V. (Hrsg.) (2010 a), Verkleinerung der hessischen Kommunalparlamente-Der Weg zur effizienteren politischen Führung, Wiesbaden.

Bund der Steuerzahler Hessen e. V. (Hrsg.) (2010 b), Kommunalparlamente jetzt verkleinern! (Pressemeldung von 6.1.2010), http://www.steuerzahler-hessen.de/Kommunalparlamente-jetzt-verkleinern/26907c30465i1p214/index.html (Zugriff: 20.7.2011).

Burth, Andreas/Gnädinger, Marc/Grieger, Thomas/Hilgers, Dennis (2012), Lexikon der öffentlichen Haushalts- und Finanzwirtschaft, Taunusstein.

Burth, Andreas/Hilgers, Dennis (2011), Finanzstatistische Analyse der Gewerbesteuer in Deutschland, in: Verwaltung \& Management, Heft 2/2011, S. 83-87.

Cohen, Jacob (1988), Statistical power analysis for the behavioral sciences, 2. Aufl., Hillsdale.

Cohen, Jacob/Cohen, Patricia/West, Stephen G./Aiken, Leona S. (2003), Applied multiple regression/correlation analysis for the behavioral sciences, Mahwah.

Crain, Mark W./Muris, Timothy J. (1995), Legislative Organization of Fiscal Policy, in Journal of Law and Economics, 38. Jg., S. 311-333.

Cusack, Thomas R. (1997), Partisan politics and public finance: Changes in public spending in the industrialized democracies, 1955-1989, in: Public Choice, 91. Jg., S. 375-395.

Deutscher Landkreistag (Hrsg.) (2011), Reformdruck für Kommunalfinanzen besteht weiter, http://www.kreise.de/ $\mathrm{cms} 1 /$ themen/kreisfinanzen/lage-der-kommunalfinanzen.html (Zugriff: 22.7.2011).

Deutscher Städte- und Gemeindebund (Hrsg.) (2011), Haushaltslage der Kommunen bleibt angespannt, http:// www.dstgb.de/dstgb/Schwerpunkte/Haushaltslage\%20der\%20Kommunen $\% 20$ bleibt $\% 20$ angespannt/Aktuelles/Haushaltslage\%20der\%20Kommunen\%20bleibt\%20angespannt/ (Zugriff: 22.7.2011).

Fahrmeier, Ludwig/Kneib, Thomas/Lang, Stefan (2009), Regression: Modelle, Methoden und Anwendungen, 2. Aufl., Berlin/Heidelberg.

Gilligan, Thomas W./Matsusaka, John G. (1995), Deviations from Constraints on Partisan Bias under the Efficient Gerrymander, in: Economic Inquiry, 33. Jg., Heft 3, S. 383-401. 
Gilligan, Thomas W./Matsusaka, John G. (2001), Fiscal Policy, Legislature Size, and Political Parties: Evidence from State and Local Governments in the First Half of the 20th Century, in: National Tax Journal, 54. Jg., Heft 1, S. 57-82.

Gnädinger, Marc (2010), Schuldenfreie Kommunen 2010 - Ein Überblick über die schuldenfreien Gemeinden und Gemeindeverbände der dreizehn deutschen Flächenländer, Taunusstein.

Gnädinger, Marc (2011), Neue Regeln für die Kommunalschuldenbremse(n) in Deutschland, in: Öffentliche Verwaltungen - Verschuldungsfähigkeit und Wirkungsorientierung, hrsg. von Reinbert Schauer, Linz, S. 55-86.

Hawkins, Douglas M. (1980), Identification of Outliers, London.

Heinemann, Friedrich/Feld, Lars P./Geys, Benny/Gröpl, Christoph/Hauptmeier, Sebastian/Kalb, Alexander (2009), Der kommunale Kassenkredit zwischen Liquiditätssicherung und Missbrauchsgefahr - ZEW Wirtschaftsanalysen (Band 93), Baden-Baden.

Hessisches Ministerium der Finanzen (Hrsg.) (2008), Hessisches Sonderinvestitionsprogramm ,Schul- und Hochschulbau“, http://www.hmdf.hessen.de/irj/servlet/prt/portal/prtroot/slimp.CMReader/HMdF_15/HMdF_Internet/med/d8b/d8b301b3-8476-0721-f012-f31e2389e481,22222222-2222-2222-2222-222222222222, true (Zugriff: 22.7.2011).

Junkernheinrich, Martin/Micosatt, Gerhard (2008), Kommunaler Finanz- und Schuldenreport Deutschland 2008 Ein Ländervergleich, Gütersloh.

Le Maux, Benoît/Rocaboy, Yvon/Goodspeed, Timothy (2011), Political fragmentation, party ideology and public expenditures, in: Public Choice, 147. Jg., S. 43-67.

Mühlenkamp, Holger/Magin, Christian (2010), Zum Eigenkapital von Gebietskörperschaften - populäre Irrtümer und Missverständnisse, in: Der Gemeindehaushalt, 111. Jg., Heft 1/2010, S. 8-11.

Müller-Marqués Berger, Thomas/Krebs, Uwe (2010), Der kommunale Gesamtabschluss - Zielsetzung, Grundlagen und Erstellung, Stuttgart.

Muzzio, Douglas/Tompkins, Tim (1989), On the Size of the City Council: Finding the Mean, in: Proceedings of the Academy of Political Science, 37. Jg., Heft 3, S. 83-96.

Nierhaus, Michael/Gebhardt, Ihno (1999), Zur Ausfallhaftung des Staates für zahlungsunfähige Kommunen, Berlin.

Pettersson-Lidbom, Per (2004), Does the size of the legislature affect the size of government? Evidence from two natural experiments, Vatt Discussion Papers No. 350, Helsinki.

Pflaumer, Peter/Heine, Barbara/Hartung, Joachim (2005), Statistik für Wirtschafts- und Sozialwissenschaften. Deskriptive Statistik, 3. Aufl., München.

Rehm, Hannes/Tholen, Michael (2008), Kommunalverschuldung - Befund, Probleme, Perspektiven, Berlin.

Rubin, Allen (2009), Statistics for Evidence-Based Practice and Evaluation, 2. Aufl., Belmont.

Statistische Ämter des Bundes und der Länder (Hrsg.) (2010), Hebesätze der Realsteuern 2009 (Gemeinschaftsveröffentlichung), Düsseldorf.

Statistisches Bundesamt (Hrsg.) (2009), Rechnungsergebnisse der kommunalen Haushalte 2007 - Fachserie 14 Reihe 3.3, Wiesbaden.

Statistisches Bundesamt (Hrsg.) (2010 a), Rechnungsergebnisse der kommunalen Haushalte 2008 - Fachserie 14 Reihe 3.3, Wiesbaden.

Statistisches Bundesamt (Hrsg.) (2010 b), Vierteljährliche Kassenergebnisse des öffentlichen Gesamthaushalts, 1.-4. Vierteljahr 2009 - Fachserie 14 Reihe 2, Wiesbaden.

Statistisches Bundesamt (Hrsg.) (2010 c), Schulden der öffentlichen Haushalte 2009 - Fachserie 14 Reihe 5, Wiesbaden.

Statistisches Bundesamt (Hrsg.) (2011), Vierteljährliche Kassenergebnisse des öffentlichen Gesamthaushalts, 1.-4. Vierteljahr 2010 - Fachserie 14 Reihe 2, Wiesbaden.

Tullock, Gordon (1959), Problems of Majority Voting, in: Journal of Political Economy, 67. Jg., Heft 6, S. 571-579.

Yates, Christopher St. John (1992), A House of Our Own or A House We've Outgrown? An Argument for Increasing the Size of the House of Representatives, in: Columbia Journal of Law and Social Problems, 25. Jg., Heft 2, S. 157-196.

Zimmermann, Horst (2009), Kommunalfinanzen - Eine Einführung in die finanzwissenschaftliche Analyse der kommunalen Finanzwirtschaft, 2. Aufl., 2009.

\section{Gesetzestexte}

Gemeindeordnung für Baden-Württemberg v. 24. Juli 2000 (GBl. 2000, S. 581), zuletzt geändert durch Gesetz v. 9. November 2010 (GB1. S. 793, 962).

Gemeindeordnung für das Land Sachsen-Anhalt v. 10. August 2009 (GVB1. LSA 2009, S. 383), zuletzt geändert durch Gesetz v. 20. Januar 2011 (GVB1. LSA, S. 14, 18).

Gemeindeordnung für den Freistaat Bayern v. 22. August 1998 (GVB1 S. 796, BayRS 2020-1-1-I), zuletzt geändert durch Gesetz v. 27. Juli 2009 (GVB1 S. 400). 
Gemeindeordnung für den Freistaat Sachsen v. 18. März 2003 (SächsGVB1. 2003, S. 55), zuletzt geändert durch Gesetz v. 7. November 2007 (SächsGVB1. 2007, S. 478).

Gemeindeordnung Rheinland-Pfalz v.31. Januar 1994 (GVB1 1994, S. 153), zuletzt geändert durch Gesetz v. 20. Oktober 2010 (GVB1. 2010, S. 319).

Gesetz über die Kommunalwahlen im Land Brandenburg v. 9. Juli 2009 (GVB1.I/09, S. 326).

Gesetz über die Wahlen in den Gemeinden und Kreisen in Schleswig-Holstein v. 19. März 1997 (GVOB1. 1997, S. 151), zuletzt geändert durch Gesetz v. 16. September 2009 (GVOB1. 2009, S. 572).

Hessische Gemeindeordnung v. 7. März 2005 (GVB1. I 2005, S. 142), zuletzt geändert durch Gesetz v. 24. März 2010 (GVB1. I S. 119).

Hessische Landkreisordnung v. 7. März 2005 (GVB1. I 2005, S. 183), zuletzt geändert durch Gesetz v. 24. März 2010 (GVB1. I 2010, S. 119, 120).

Kommunalselbstverwaltungsgesetz Saarland v. 27. Juni 1997 (Amtsbl., S. 682), zuletzt geändert durch Gesetz v. 11. Februar 2009 (Amtsbl., S. 1215).

Kommunalwahlgesetz für das Land Mecklenburg-Vorpommern v. 13. Oktober 2003 (GVOBl. M-V 2003, S. 458), zuletzt geändert durch Gesetz v. 16. Dezember 2010 (GVOB1. M-V 2010, S. 690).

Kommunalwahlgesetz Nordrhein-Westfalen v. 30. Juni 1998 (GV. NRW, S. 384), zuletzt geändert durch Gesetz v. 3. Mai 2011 (GV. NRW, S. 238).

Landkreisordnung für Baden-Württemberg v. 19. Juni 1987 (GB1. 1987, S. 288), zuletzt geändert durch Gesetz v. 9. November 2010 (GB1. 2010, S. 793, 962).

Landkreisordnung für das Land Sachsen-Anhalt v. 12. August 2009 (GVB1. LSA 2009, S. 435), zuletzt geändert durch Gesetz v. 20. Januar 2011 (GVB1. LSA S. 14, 18).

Landkreisordnung für den Freistaat Bayern v. 22. August 1998 (GVB1 S. 826, BayRS 2020-3-1-I), zuletzt geändert durch Gesetz v. 27. Juli 2009 (GVB1 S. 400).

Landkreisordnung für den Freistaat Sachsen v. 19. Juli 1993 (SächsGVBl. 1993, S. 577), zuletzt geändert durch Gesetz v. 7. November 2007 (SächsGVB1. 2007, S. 478, 482).

Landkreisordnung Rheinland-Pfalz v. 31. Januar 1994 (GVBl 1994, S. 188), zuletzt geändert durch Gesetz v. 20. Oktober 2010 (GVB1. 2010, S. 319).

Niedersächsische Gemeindeordnung v. 28. Oktober 2006 (Nds. GVB1. 2006, S. 473), zuletzt geändert durch Gesetz v. 17. Dezember 2010 (Nds. GVB1. 2010, S. 576).

Niedersächsische Landkreisordnung v. 30. Oktober 2006 (Nds. GVB1. 2006, S. 510), zuletzt geändert durch Gesetz v. 17. Dezember 2010 (Nds. GVB1. 2010, S. 576).

Thüringer Kommunalordnung v. 28. Januar 2003 (GVB1. 2003, S. 41), zuletzt geändert durch Gesetz v. 22. Juni 2011 (GVB1. 2011, S. 99, 134). 